University of Nebraska - Lincoln

DigitalCommons@University of Nebraska - Lincoln

2012

\title{
A Social Network Perspective on Measurements of Dominance Hierarchies
}

Daizaburo Shizuka

University of Nebraska-Lincoln, dshizuka2@unl.edu

David B. McDonald

University of Wyoming, dbmcd@uwyo.edu

Follow this and additional works at: https://digitalcommons.unl.edu/bioscifacpub

Shizuka, Daizaburo and McDonald, David B., "A Social Network Perspective on Measurements of Dominance Hierarchies" (2012). Faculty Publications in the Biological Sciences. 234.

https://digitalcommons.unl.edu/bioscifacpub/234

This Article is brought to you for free and open access by the Papers in the Biological Sciences at DigitalCommons@University of Nebraska - Lincoln. It has been accepted for inclusion in Faculty Publications in the Biological Sciences by an authorized administrator of DigitalCommons@University of Nebraska - Lincoln. 


\title{
A Social Network Perspective on Measurements of Dominance Hierarchies
}

\author{
Daizaburo Shizuka and David B. McDonald \\ Department of Zoology and Physiology, University of Wyoming, Laramie, WY, USA \\ Corresponding author - D. Shizuka
}

\begin{abstract}
The hierarchical organization of dominance relations among animals has wide-ranging implications in social evolution. The structure of dominance relations has often been measured using indices of linearity (e.g. Landau's $h$, Kendall's K): the degree to which dominance relations adhere to a linear hierarchy. An alternative measure is the transitivity of dominance relations among sets of three players that all interact with each other, a measure we call triangle transitivity $\left(t_{\text {tri }}\right)$. Triangle transitivity and linearity are essentially equivalent when dominance relations of all dyads are known, but such complete observations are rare in empirical studies. Triangle transitivity has two major advantages: it does not require 'filling in' of unobserved relations, and its expected value is constant across group sizes. We use a social network perspective to demonstrate a property of transitivity in random directed networks (on average, three-fourths of complete triads are transitive) and show that empirical dominance networks are often significantly more transitive than random networks. Using 101 published dominance matrices we show that published algorithms for assessing linearity underestimate the level of social orderliness, particularly in larger groups, which tend to have more null dyads. Thus, previous puzzlement over the decrease in estimated linearity in larger groups could be due largely to the bias introduced by random filling of null dyads. We argue that triangle transitivity will allow researchers to focus on important processes underlying the dynamics of dominance, such as spatial segregation, avoidance of interactions by certain individuals and detailed temporal patterns in the ontogeny of hierarchy formation.
\end{abstract}

Keywords: aggression, imputation, Landau's $h$, orderliness, peck order, random network, social structure, triad census

The existence of hierarchical order in social systems is a general and striking pattern in nature (Dawkins 1976). A primary example is the prevalence of dominance hierarchies, in which the members of a group establish dominance ranks that influence access to resources or mating opportunities (Drews 1993). How social order on the scale of whole societies or groups can emerge from a series of social interactions between two individuals has been a central question in social biology (Landau, 1951a, 1951b; Chase, 1982a; Dugatkin, 1997; Faust, 2007). An important component of such research is to establish useful metrics that can capture essential aspects of hierarchical organization.

Since the first descriptions of 'peck order' in groups of hens (Schjelderup-Ebbe 1922), studies of dominance structure have often focused on why animal groups often seem to be arranged into linear hierarchies. A strictly linear hierarchy is one in which higher-ranked individuals dominate all individuals of lower rank. Within a strictly linear hierarchy, all dyads have a dominant-subordinate relation, and dominance relations for every set of three players (triads) are 'transitive': when individual $A$ dominates $B$ and $B$ dominates $C$, then $A$ also dominates C (Chase, 1982a; de Vries, 1995). In contrast, a triad arranged in a 'cycle' (e.g. A dominates B, B dominates $\mathrm{C}$, and $\mathrm{C}$ dominates $\mathrm{A}$ ) results in dominance relations that are unre- solved and prevents the linear arrangement of ranks. The orderly and predictable arrangement of dominance ranks in a linear hierarchy is thought to have important consequences for individual fitness and group stability (Ellis, 1995; van Doorn et al., 2003; Cant et al., 2006). The structure of dominance hierarchies is typically measured using one of two independently derived indices, Kendall's $K$ and Landau's $h$ (Kendall and Babington Smith, 1940; Landau, 1951a; Appleby, 1983; de Vries, 1995; also see Methods). Although they are not identical, the two indices generally yield the same values (see Methods; de Vries 1995) describing the degree to which an animal group adheres to a strict linear hierarchy. Thus, these indices are commonly referred to as metrics of 'linearity'.

A major limitation of linearity indices is that they become biased when some pairs of individuals fail to interact (de Vries, 1995; Klass and Cords, 2011). This bias arises in part because strict linear hierarchies can occur only in groups where dominant-subordinate relations exist for every dyad in the group. Networks in which all dyadic relations are asymmetrical (e.g. dominant-subordinate) are called 'tournaments' (Harary \& Moser 1966). However, tournaments rarely arise naturally in animal groups, and as we will show, unknown dyadic relationships are common because certain pairs of individuals fail to interact (hereafter 'null dyads'). Currently popular 


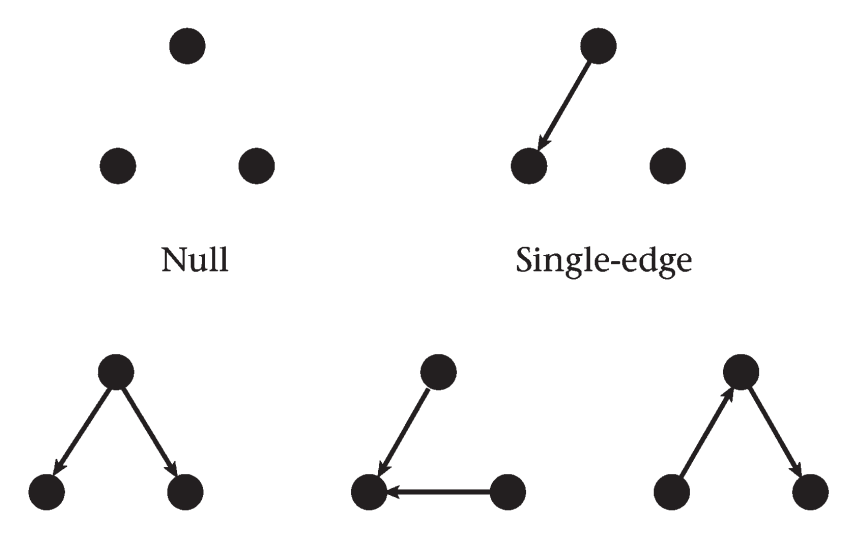

Double-dominant Double-subordinate Pass-along

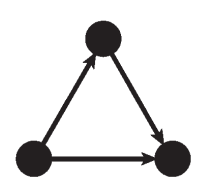

Transitive

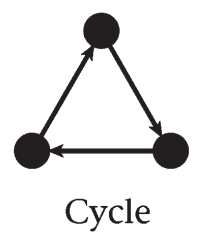

Figure 1. Configurations of seven possible triads in a network with no mutual dyads. Triangles (triads with three edges) can either be 'Transitive' or 'Cyclic'.

methods call for random 'imputation': null dyads (missing data) are 'filled in' with randomized dominance relations (de Vries 1995). Randomization procedures are often used to reduce bias in such imputation methods (Nakagawa \& Freckleton 2008). However, we show that random filling of null dyads inevitably produces a different but pernicious negative bias in estimated linearity.

Dyads may fail to interact for multiple reasons, and the processes that lead to noninteractions may be of biological interest. First, spatial or temporal segregation may make it impossible for some dyads to interact. Inferring a dominance relation between such individuals would be a purely artificial exercise. Alternatively, some individuals may actively avoid interactions. Such avoidance could arise because the costs of interaction are too high (e.g. escalation of contest is likely), the potential benefits of interaction are too low, or because dominance relations can be settled without resorting to overt interactions (e.g. by long-distance signalling or by third-party effects). Finally, some interactions may simply have been unobserved. It is difficult and often impossible to distinguish between these processes that give rise to noninteractions. However, it is possible to measure directly the frequency of transitive and cyclic triadic relations within the observed set of dominance interactions, thereby avoiding the bias introduced by imputation procedures. Using this approach, we reveal heretofore-unappreciated levels of orderliness in animal groups.

Our approach is based on the triad census, a tool commonly used in social network analysis to count directly the frequencies of all triadic configurations in the data (Figures 1 \& A1; Holland and Leinhardt, 1970, 1976; Wasserman and Faust, 1994; Faust, 2007). The triad census allows us simply to compare the frequencies of transitive and cyclic triads. The relative frequencies of triadic configurations, or 'motifs' are routinely used to characterize the structural properties of networks (Kendall and Babington Smith, 1940; Harary and Moser, 1966; Holland and Leinhardt, 1976; Frank and Harary, 1982; Karl-
Table 1. Operational definitions of terms

$\mathrm{Cycle}^{*}$ - A particular form of triad in which directional relations form a cycle, e.g. A dominates $\mathrm{B}, \mathrm{B}$ dominates $\mathrm{C}$, and $\mathrm{C}$ dominates $\mathrm{A}$ $(\mathrm{A}>\mathrm{B}>\mathrm{C}>\mathrm{A})$. In a tournament, all intransitive triads are cycles.

Imputation - A general term for procedures to substitute a missing data point with some value.

Linear hierarchy - Dominance structure fulfilling two criteria: (1) all dyads have a dominant-subordinate relation, and (2) all triadic relations are transitive. Synonyms: complete acyclic digraph, peck order, transitive tournament.

Linearity - A generic term to describe one of two indices: Kendall's $K$ and Landau's $h$. These both describe how close the structure of dominance relation is to a linear hierarchy. See Methods for details on the indices.

Orderliness - The tendency of triadic relationships to be ordered, or transitive. By our definition, linearity and triangle transitivity are both measures of orderliness.

Tournament - A directed network composed purely of asymmetrical dyads. Synonym: Round robin tournament.

Transitive triad* - A set of three nodes that are all connected to each other, in which the asymmetrical relationships are transitive (if $\mathrm{A}>\mathrm{B}$ and $\mathrm{B}>\mathrm{C}$, then $\mathrm{A}>\mathrm{C}$ ). Synonyms: ordered triple.

Transitivity* $^{*}$ Generically, the proportion of a given set of triads that are transitive. Mathematically, it can be defined in several different ways for directed and undirected networks (e.g., see Holland and Leinhardt, 1976; Frank and Harary, 1982; Karlberg, 1999).

Triangle - A set of three nodes, each of which is connected to the other two. Synonym: closed triad, complete triad.

Triangle Transitivity - An index based on the proportion of transitive triangles among all triangles in a network.

* These terms have different definitions when applied to undirected networks.

berg, 1999; Milo et al., 2002; Faust, 2008; Allesina and Levine, 2011). Here, we build on this body of work to define a metric $\left(P_{t}\right)$ that quantifies the proportion of transitive triads among all 'triangles' (those triads in which all dominance relations were established and thus could potentially be transitive). We will first show that the expectation for $P_{t}$ in a random network is 0.75 , regardless of group size or matrix sparseness. Using this expectation as the baseline, we develop a scaled index of 'triangle transitivity' $\left(t_{\text {tri }}\right)$. Measures of linearity and triangle transitivity take different approaches to describe an essential component of dominance hierarchies: the tendency of triadic relations to be ordered (i.e. transitive) rather than cyclic. Linearity ( $K$ and $h$ indices) describes the transitivity of dominance relations in the context of tournaments, whereas triangle transitivity $\left(t_{\text {tri }}\right)$ is the transitivity of the subset of triads in which all dominance relations were observed. These both differ slightly from other measures of transitivity ( Holland and Leinhardt, 1976; Frank and Harary, 1982; Karlberg, 1999). Because the terms 'linearity', 'transitivity' and 'triangle transitivity' all refer to distinct methods for measuring the tendency of triadic relations to be ordered (Table 1), we will use the general term 'orderliness' to refer to this family of metrics. Our goals are to use a network approach to illuminate some complications related to the analysis of orderliness in dominance relations in animal groups and provide some potential avenues for further exploration of social systems.

\section{Methods}

\section{Calculating Measures of Linearity}

de Vries (1995) gave a thorough account of two methods of measuring linearity: Kendall's $K$ (originally denoted as $\zeta$ : Kendall and Babington Smith, 1940; Appleby, 1983) and Landau's $h$ (Landau 1951a). With the exception of subtle differences when measuring linearity in groups with even versus odd 
numbers of individuals, these two values are nearly identical. Because most current empirical studies of dominance structure use a modified version of Landau's method (denoted $h^{\prime}$ : de Vries 1995), we will focus on comparisons between $h^{\prime}$ and our metric $t_{\text {tri }}$. Nevertheless, an overview of the earlier linearity indices is instructive for purposes of distinguishing them from our index of triangle transitivity.

Kendall's $K$ is defined as the proportion of cyclic triads observed (d), relative to the maximum possible number of cycles (Kendall \& Babington Smith 1940). For a group of $N$ individuals, the dominant-subordinate relations are summarized in a $N \times N$ matrix of dominance scores. For convenience, we will use Appleby's (1983) criteria for scoring in the dominance matrix. Individuals that win more than $50 \%$ of encounters in each dyad receive a score of 1 in its row at the column position of the subordinate. If both individuals win an equal number of encounters, each receives a score of 0.5 in its respective row-column positions. Other criteria for assigning dominance scores are possible. The most appropriate criteria depend on the research subjects and the questions addressed. For each individual $i, S_{i}$ is the row sum (dominance total) of these scores. Subordinance totals are given by the column sums. Kendall \& Babington Smith (1940) showed that the number of cyclic triads is

$$
d=\frac{N(N-1)(2 N-1)}{12}-1 / 2 \sum\left(S_{i}\right)^{2}
$$

The maximum number of cyclic triads possible among $N$ individuals is

$$
\begin{cases}d_{\max }=1 / 24\left(N^{3}-N\right) & \text { for odd values of } N \\ d_{\max }=1 / 24\left(N^{3}-4 N\right) & \text { for even values of } N\end{cases}
$$

Given these,

$$
K=1-\frac{d}{d_{\max }}
$$

Thus, $K$ is an index of the frequency of cyclic triads relative to the maximum possible. $K=1$ when no cyclic triads exist, and $K=0$ when the number of cyclic triads is maximal. Note that the dependence of the measure on whether $N$ is odd or even makes this measure problematic for comparing linearity across groups of differing size.

Landau's (1951a) approach is based on the variance in dominance among individuals. Maximum variance occurs when the hierarchy is completely linear. Using the same notation as above, Landau's (1951a) hierarchy index is defined as

$$
h=\frac{12}{N^{3}-N} \sum_{i=1}^{N}\left[S_{i}-\frac{N-1}{2}\right]^{2}
$$

The value of $h$ also ranges from 0 to 1 , with $h=1$ being perfect linearity. Values of $K$ and $h$ are equal for odd-sized groups but differ slightly for even-sized groups.

Both Kendall's $K$ and Landau's $h$ are derived from analyses of tournaments. When null dyads occur, $K$ and $h$ are biased towards underestimating linearity (Appleby, 1983; de Vries, 1995). Currently, a randomization procedure is commonly used whereby dominant (1) versus subordinate (0) scores are randomly imputed to the members of each null dyad. An $h$ value is calculated for this 'filled' dominance matrix, and the modified Landau's $h$ (denoted as $h^{\prime}$ ) is the average $h$ value of 10000 randomly filled matrices (de Vries 1995). This is also the first randomization step in the statistical test advocated by de Vries (1995), which is described later (see Statistical Test of Linearity and Triangle Transitivity).

\section{Calculating the Index of Triangle Transitivity, $t_{\text {tri }}$}

Our method for measuring triangle transitivity, $t_{\text {tri }}$ is based on direct enumeration of the triad types without resorting to randomly filling in null dyads. The matrix of dyadic dominance relations is directly equivalent to a network adjacency matrix (Wasserman \& Faust 1994), with the slight modification that when two individuals win the same number of contests against each other, they both receive scores of 1 instead of 0.5 . In the corresponding network diagram, a dominant-subordinate relation (asymmetric dyad) is represented by an edge (arrow) directed from the dominant to the subordinate node (individual). Null dyads lack a connecting edge. Mutual dyads (two-way arrows), in which both individuals win the same number of contests, are very rare in dominance data sets (on average, $2.2 \%$ of dyadic relations were mutual in our sample of 101 empirical networks; Supplementary material, Table S1). Moreover, mutual dyads are necessarily transient states, and can occur only when the dyad has interacted an even number of times. From a methodological standpoint, mutual dyads and null dyads are similar: they both represent an unresolved dyadic relation such that one additional interaction would create an asymmetrical dyad. A more comprehensive treatment of the transitive dynamics of dominance relation would need to account for such complications, but this is beyond the scope of the current study. Here, we first discuss the measurement of triangle transitivity in networks that contains no mutual dyads. We do so because it results in a greatly simplified triad census (Holland \& Leinhardt 1976) of seven rather than 16 types, without affecting any of our conclusions. This will allow us to present our approach in its simplest form. However, the approach can be extended to include networks with mutual dyads, and we discuss the calculation of the triangle transitivity in networks with mutual dyads in the Appendix.

A directed network with only asymmetric or null dyads has seven distinct (nonisomprphic) types of triads (Figure 1; see Figure A1 for the 16 types possible with mutual edges; Holland and Leinhardt, 1976; Wasserman and Faust, 1994). Each combination of three nodes (e.g. 165 triads in an 11-animal group) is distributed across these seven possible types. The triad census is implemented in network analysis packages in R (e.g. statnet: Handcock et al. 2003; igraph: Csárdi \& Nepusz 2006). Of the seven triad types, we focus particularly on the two triangles (i.e. triads connected by three edges): the transitive and cyclic triangles. The proportion of transitive triangles relative to all triangles $\left(P_{t}\right)$ is given by

$$
P_{t}=\frac{N_{\text {transitive }}}{N_{\text {transitive }}+N_{\text {cycle }}}
$$

where $N_{\text {transitive }}$ is the number of transitive triangles, and $N_{\text {cycle }}$ is the number of cyclic triangles. Codes for calculating $P_{t}$ using the statnet package (Handcock et al. 2003) in R (R Development Core Team 2009) are presented in the Supplementary material.

In random networks, $P_{t}$ is expected to equal 0.75 . This property can be demonstrated intuitively by considering all possible triangle configurations between three individuals A, B and C (Figure 2; Appleby 1983). Of eight possible configurations, six, or $75 \%$, are transitive. A mathematical derivation, based on the expected frequencies for random networks (Holland \& Leinhardt 1976) is given in the Appendix. Furthermore, the result is easily shown by simulation of a large sample of random networks and calculating $P_{t}$.

With an expected value of $P_{t}=0.75$, we can scale transitivity so that it runs from 0 for the random expectation to 1 (all triangles are transitive, no cycles). Our triangle transitivity metric, $t_{\text {tri }}$ is then given by 

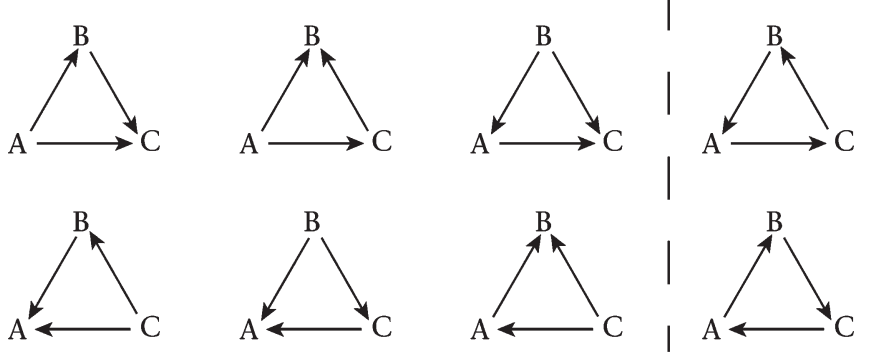

Transitive

Cyclical

Figure 2. All eight possible patterns of dominant-subordinate relations between individuals A, B and C. Triangles to the left of the dashed line are transitive and those on the right are cyclic. Six of eight, or $75 \%$, of possible triangles are transitive.

$$
t_{\text {tri }}=4\left(P_{t}-0.75\right)
$$

Note that $t_{\text {tri }}$ could be negative if more cyclic triangles occurred than would be expected in a random network. Empirical data sets rarely have $t_{\text {tri }}$ values that are negative, and even then the values are always close to 0 (Supplementary Table S1).

Triangle transitivity, $t_{\text {tri, }}$ can also be calculated for each iteration of the 'random fill' process as described by de Vries (1995; see Calculating Measures of Linearity). We will refer to the average transitivity from these 'filled' networks as $t_{\text {tri. }}$. Comparing $h^{\prime}$ and $t^{\prime}$ tri allows us to directly compare the value of linearity and transitivity in imputed networks. Comparing values of $t^{\prime}$ tri from imputed matrices and $t_{\text {tri }}$ from the raw data allows us to disentangle the effect of the imputation procedure itself on measures of dominance structure.

\section{Statistical Analysis of Linearity and Triangle Transitivity}

A two-step randomization procedure advocated by de Vries (1995) is the predominant statistical test of significant linearity. In the first step, one imputes a random dominance relation to all null dyads and then calculates an initial linearity, $h_{0}$. In the second step, all dyadic dominant-subordinate relations are randomized. The linearity of this randomized matrix is $h_{r}$. These two values, $h_{0}$ and $h_{r^{\prime}}$ are compared with each other. Steps 1 and 2 are repeated 10000 times; the one-tailed $P$ value is the proportion of randomizations for which $h_{r} \geq h_{0}$. In essence, this procedure compares the linearity of an empirical, although imputed, matrix with the expected linearity from random matrices of the same size. The average value of $h_{0}$ from the 10000 simulations is the $h^{\prime}$ index (see Calculating Measures of Linearity).

To assess the significance of our transitivity metric we use logic similar to that of de Vries (1995), but the procedure is conducted without imputing null dyads. In network theory, this is called a conditional uniform graph approach (Wasserman and Faust, 1994; Faust, 2010). First, we generate 1000 dyad census-conditioned random graphs; these random networks simulate the dominance structure for hypothesized groups of the same number of individuals and same number of observed dominance relationships, but with uniform probability for each individual dominating any other individual. We then compare the range of triangle transitivity values in these random graphs $\left(t_{\text {random }}\right)$ to the empirical value, $t_{\text {tri }}$. The one-tailed $P$ value is the proportion of times $t_{\text {random }} \geq$ empirical $t_{\text {tri }}$. The application of this procedure for networks with mutual dyads is discussed in the Appendix. In the Supplementary material, we provide the codes for conducting this procedure using the statnet package (Handcock et al. 2003) in $R(R$ Development Core Team 2009).

\section{Comparing Transitivity and Linearity in Random Networks}

To assess the effects of network size on measures of linearity and transitivity, we simulated random tournaments (i.e. networks composed entirely of asymmetrical dyads) of varying sizes (range 5-50 nodes), with 1000 replicates for each network size. For replicate random tournaments of a given size, we measured the mean and confidence intervals of $h$ and $P_{t}$. Note that in tournaments, there are no null dyads to impute, so $h^{\prime}=h$. Similarly, in tournaments, $P_{t}=1$ represents a completely linear hierarchy because all dyads have a dominantsubordinate relationship, and all dominance relations are transitive. To determine the range of possible $P_{t}$ values, we also calculated the minimum value of $P_{t}$ possible in each set of simulations using

$$
P_{t \text { min }}=1-\frac{d_{\text {max }}}{N_{\text {transitive }}+N_{\text {cycle }}}
$$

where $d_{\max }$ is the maximum number of cyclic triangles, as defined by equation (2). This calculation only applies to tournaments (Kendall \& Babington Smith 1940), as we do not know of any algorithm for calculating the minimum number of transitive triads in sparse networks.

We also investigated the effect of network sparseness (proportion of dyads that are null) on values of $P_{t}$ by constructing random networks of constant size (20 nodes) but of varying sparseness (range 0-0.9), and plotting the means and confidence intervals of $P_{t}$ for 1000 replicates of each level of network sparseness. To match the one-tailed statistical test of linearity and triangle transitivity, our confidence interval represents 0 to 95 th percentile of $h$ and $t_{\text {tri }}$ values in all of our random networks. Values that are outside this range would be considered statistically significant at $\alpha=0.05$.

\section{Comparing Transitivity and Linearity in Empirical Networks}

We collected 101 matrices containing raw win-loss totals from 55 studies published in peer-reviewed journals (Supplementary Table S1). These included studies on invertebrates, fish, birds and mammals, with group size ranging from 6 to 45 individuals. We excluded matrices of five or fewer individuals, and those with fewer than two interactions per individual. Some studies included multiple matrices (either observations of different groups or of the same group at different times), and we included all available matrices that fit our criteria. Although the sample is not exhaustive, it covers a wide range of taxa and hierarchical structures. We first analyse empirical networks in which we exclude mutual dyads because they are rare and pose theoretical complications (see Calculating the Index of Triangle Transitivity above). In the Appendix, we present the method of calculating triangle transitivity in networks with mutual dyads, and show that excluding mutual dyads has negligible effects on the measure of triangle transitivity.

We calculated linearity and triangle transitivity indices from randomly filled dominance matrices $\left(h^{\prime}\right.$ and $t^{\prime}{ }_{\text {tri }}{ }^{\prime}$ respectively), using the procedure described in de Vries (1995; see "Calculating Measures of Linearity" and "Calculating the Index of Triangle Transitivity," above). We implemented the randomization routine in $\mathrm{R}$. We then calculated the scaled index of triangle transitivity $\left(t_{\text {tri }}\right)$ from empirical matrices using only the raw data presented in the publications (see Supplementary material for codes written in R). Finally, we calculated the $P$ values associated with statistical tests of $h^{\prime}$ and $t_{\text {tri }}$ from the empirical data using the procedure outlined above (see Statistical Test of Linearity and Transitivity). 

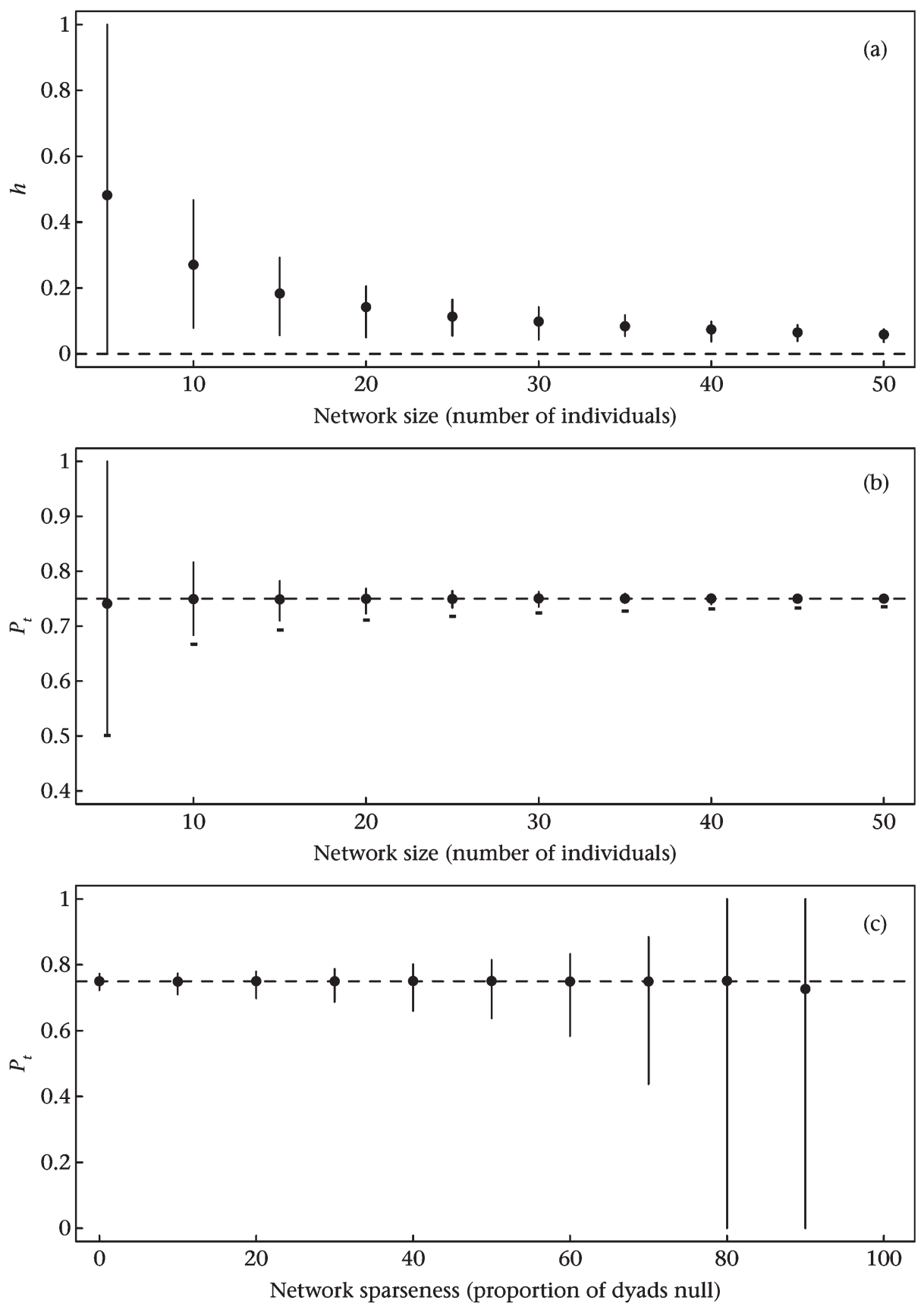

Figure 3. Values of linearity and transitivity in simulated random networks. Symbols represent mean values of (a) $h$ or (b) $P$ from 1000 simulated random tournaments of varying sizes, or (c) mean values of $P_{t}$ from 1000 simulated random networks of $N=20$ individuals and varying sparseness. We did not calculate $h$ in sparse networks. Lines represent the 0 to 95th percentile of $h$ and $P$, values for replicate simulated networks of each size or density. Dotted line represents $h=0$ in (a), and $P_{t}=0.75$ in (b, c). In (b), the horizontal bars represent the minimum level of transitivity possible in a tournament of a given size, calculated by equation (7). Average values of Landau's $h$ change with network size, but average values of $P_{t}$ do not change with network size or sparseness. Variance of both $h$ and $P_{t}$ decrease with increasing network size, and variance of $P_{t}$ also increases in networks with more null dyads. Minimum levels of transitivity in sparse networks cannot be calculated analytically. Small, sparse networks are not amenable to statistical tests of social structure because the variance in expected values is large, and even perfect linearity or transitivity would not differ significantly from random.

\section{General Statistical Methods}

We investigated the effects of network size and network sparseness on measures of linearity and triangle transitivity using linear regression. Network size was log transformed, and network sparseness was arcsine square root transformed to conform to normality assumptions for linear regression. All statistical and simulation procedures were conducted in $\mathrm{R}$.

\section{Results}

\section{Triangle Transitivity and Linearity in Random Networks}

We first evaluated how network size (number of individuals in the group) affected the random expected values of $h$ and $P_{t}$ (Figure $3 \mathrm{a}, \mathrm{b}$ ). We confirmed that network size affected the $h$ estimate of linearity in random tournaments: the expected 
values of $h$ decreased with increasing group size (Figure 3a: Landau 1951a). In contrast, the average proportion of transitive triads $\left(P_{t}\right)$ remained constant at 0.75 over various network sizes (Figure $3 b$ ). The 95\% confidence intervals shown in Figure 3 illustrate the range of $h$ and $P_{t}$ values that could arise by chance (for one-tailed tests at $\alpha=0.05$ ). The confidence interval is larger for smaller tournaments, and includes $h=1$ or $P_{t}=1$ in tournaments of five individuals, showing that perfectly linear hierarchies often arise by chance in very small groups. Similarly, the average value of $P_{t}$ also stayed constant at 0.75 at different levels of network sparseness, but the 95\% confidence interval increased when the network was very sparse (Figure 3c).

The change in expected values of $h$ also means that the interpretation of dominance structure at a given value of $h$ could vary depending on group size. For example, $h=0.4$ at $N=5$ individuals is not significantly different from the random expectation, but $h=0.4$ at $N=40$ would be considered significantly linear.

In summary, while the statistical probability of a given value of $h$ and $P_{t}$ arising by chance are both affected by network size, only $P_{t}$ serves as a reliable indicator of the sign (positive or negative) of the orderliness of dominance structure relative to random expectation. In particular, $P_{t}>0.75$ always indicates that there are more transitive triangles than the null expectation. We therefore scale our index of triangle transitivity, $t_{\text {tri }}$ (equation 6), relative to 0.75 . However, the magnitude of the $t_{\text {tri }}$ value is not necessarily informative as a statistical index in and of itself because the confidence intervals around this expected value vary with network size and density.

\section{Triangle Transitivity and Linearity in Empirical Networks}

To determine whether linearity and triangle transitivity measures yield fundamentally different estimates of social structure, we simultaneously calculated $h^{\prime}$ and $t^{\prime}{ }_{\text {tri }}$ from 10000 replicates of each of the 101 empirical data sets whose null dyads we filled randomly. The objective was to compare the indices under the same conditions of random filling. The linearity and transitivity indices were tightly correlated (Pearson's correlation: $r_{99}=0.98, N=101$ matrices, $P<0.001$ ), but the best-fit curve to explain the relationship between the two measures was nonlinear (second-degree polynomial: $y=-0.02+0$. $35 \times x+0.70 \times x^{2}$; Supplementary Figure S1). The nonlinear relationship means that the two indices are not interchangeable. The nonlinearity is most likely the result of how the values of the two indices differ in response to changing network size (Figure 3a, b). To support this claim, linear regression with $h^{\prime}$, but with network size included as a covariate, explained $99 \%$ of the variation in the value of $t_{\text {tri }}^{\prime}\left(F_{3,97}=4286, R_{\text {adj }}^{2}=0.99\right.$, $P<0.001)$, a significantly better fit compared to the model that did not include network size (Wald test: $F_{2,98}=254.6$, $P<0.001$; linear model without network size: $F_{1,99}=2016, R^{2}$ adj $=0.95, P<0.001)$. That result suggests that under the same constraints (i.e. when all dyads are randomly assigned a dominance relation and when network size is included as a covariate), $t_{\text {tri }}^{\prime}$ and $h^{\prime}$ are essentially equivalent measures.

We then compared triangle transitivity, $t_{\text {tri, }}$, from empirical networks with unmanipulated null dyads, with $t^{\prime}{ }_{\text {tri }}$, where null dyads were randomly filled. Comparing $t_{\text {tri }}$ and $t_{\text {tri }}$ allowed us to investigate the effect of the random-fill imputation procedure used in calculating the $h^{\prime}$ linearity index.

The triangle transitivity, $t_{\text {tri }^{\prime}}$ in the unmodified dominance data sets was greater than $t_{\text {tri }}^{{ }^{t}{ }^{\prime}}$ in the randomly filled data, indicating that the imputation procedure leads to overly conservative estimates of orderliness (mean \pm SE: $t_{\text {tri }}=0.88 \pm 0.02$; $t^{\prime}{ }_{\text {tri }}=0.53 \pm 0.03$; Wilcoxon signed-ranks test: $Z=8.2, P<0.001$;
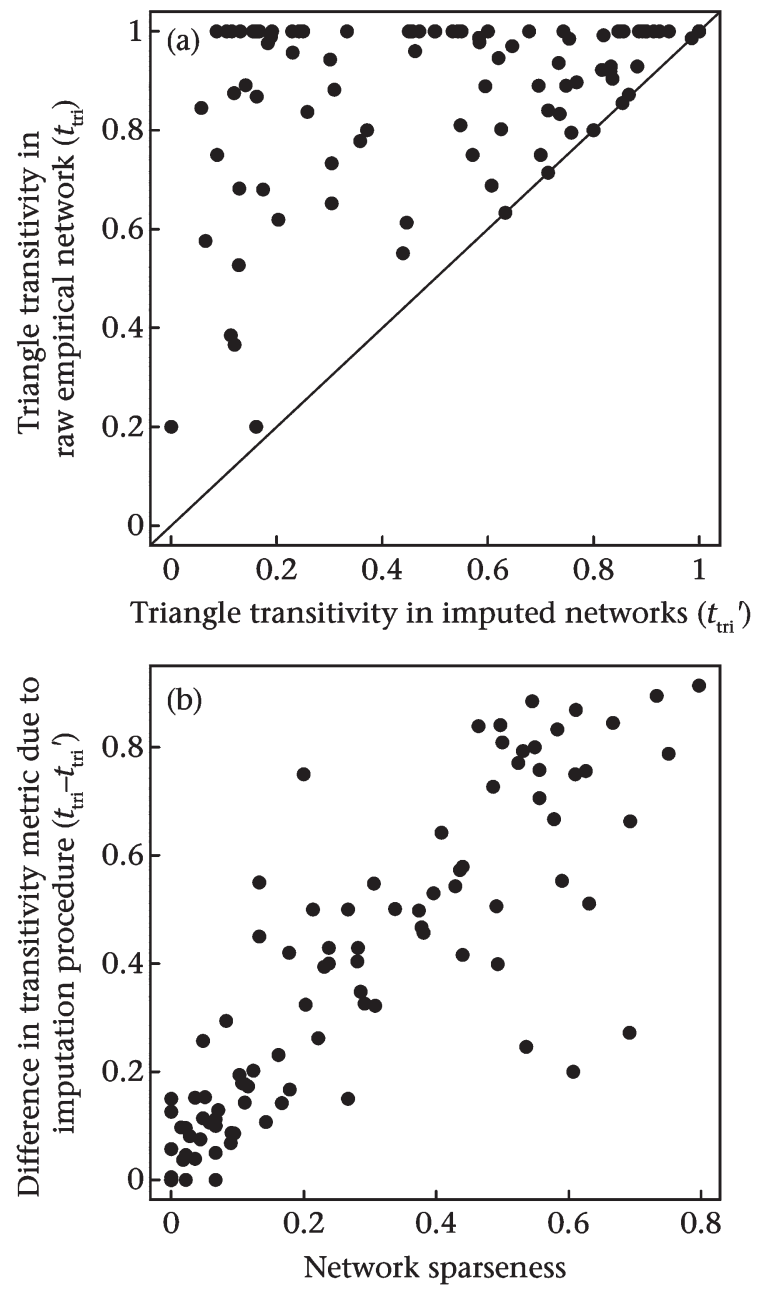

Figure 4. Randomly filling in null dyads reduces the transitivity value of social dominance networks. (a) Comparison of filled versus raw dominance networks shows that triangle transitivity in randomly filled networks $\left(t_{\text {tri }}{ }^{\prime}\right)$ is systematically lower than when triangle transitivity is calculated without manipulating the data $\left(t_{\text {tri }}\right)$. (b) Amount of discrepancy between imputed and raw networks is explained by the proportion of dyads that were imputed. Note that some overlapping data points exist.

Figure 4a). The reduction in triangle transitivity associated with imputation is related to the proportion of null dyads (linear regression: $F_{1,99}=304.1, R^{2}$ adj $=0.75, P<0.001$; Figure $4 \mathrm{~b}$ ). Of the 101 data sets, $88(87 \%)$ contained at least one null dyad, and the proportion of null dyads (mean \pm SE $=0.26 \pm 0.02$ ) was positively correlated with the number of individuals (Pearson's correlation: $r_{99}=0.46, N=101, P<0.001$ ). Moreover, $33(33 \%)$ of the 101 data sets contained no cyclic triads $\left(P_{t}=t_{\text {tri }}=1\right)$, while only four of the 101 had an $h$ value of 1 . Thus, filling in null dyads artificially creates cyclic triads and systematically underestimates orderliness. Because null dyads tend to be more abundant in studies of larger groups, the apparent decrease in linearity with increasing group size is most likely an artefact of the imputation procedure used in calculating $h^{\prime}$.

We used linear regression to confirm that group size and proportion of null dyads alone explain most of the variation in the linearity metric, $h^{\prime}$ (full model with network size and sparseness as covariates: $F_{3,100}=149.2, R_{\text {adj }}^{2}=0.82, P<0.001$ ). Specifically, estimated linearity decreases with increasing network size or sparseness (Supplementary Figure S2a, b). In contrast, our transitivity metric, $t_{\text {tri }}$, was unaffected by either 


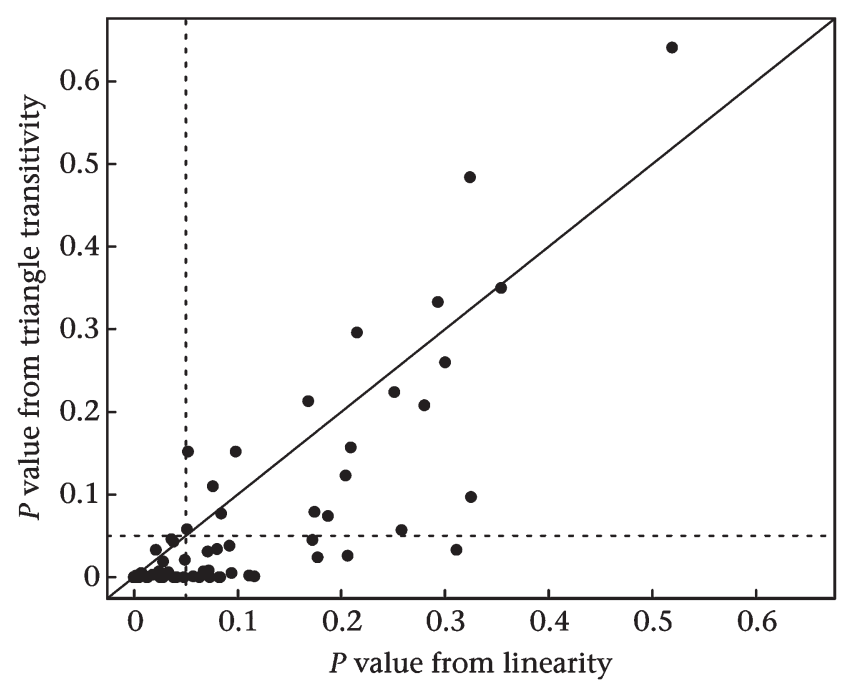

Figure 5. Comparison of $P$ values from linearity and transitivity measures. Statistical analysis of linearity followed de Vries (1995). Solid line is a 1:1 line, and dotted lines indicate $a=0.05$ for each axis. Imputed linearity often underestimates the statistical significance of social orderliness. Of 101 empirical dominance networks analysed, 15 that would have been interpreted as nonlinear actually had significant levels of transitivity at $\alpha=0.05$ (one-tailed simulation test for both). Note that some overlapping data points exist.

network size or sparseness (Supplementary Figure S2c, d; linear regression, full model with size and sparseness as covariates: $F_{3,100}=0.32, R^{2}{ }_{\text {adj }}=-0.02, P=0.81$ ). Therefore, it is probably a more unbiased measure of social orderliness that is amenable to comparisons across studies.

The imputed metric of linearity, $h^{\prime}$, could lead to various misinterpretations of social orderliness in animal groups. While the $P$ values derived from the linearity and transitivity procedures were correlated (Pearson's correlation: $r_{99}=0.85$, $N=101, P<0.001$ ), the $P$ values for linearity were larger (i.e. less significant) than those for triangle transitivity (Wilcoxon signed-ranks test: $Z=-7.4, P<0.01$; Figure 5), suggesting that imputation-based linearity underestimates the threshold for significant social orderliness. Thus, 15 of the 101 data sets in which the linearity failed to detect significant structure were deemed significantly nonrandom using triangle transitivity $(\alpha=0.05)$, whereas only one data set was significantly linear but marginally nonsignificant with triangle transitivity (Figure 5). Some studies, especially those with large, sparse matrices, might therefore conclude that groups contain no significant hierarchical structure if relying on the linearity index, $h^{\prime}$, even when the triadic relationships that actually exist are disproportionately transitive.

\section{Discussion}

In this study, we define triangle transitivity $\left(t_{\text {tri }}\right)$ as a scaled index of the relative frequency of transitive triads among all triangles (closed triads) in a dominance network. This allows us to ask the question: of the three-way dominance relationships we actually observed, what proportion of these are transitive rather than cyclic? Our method is based on the triad census, a network analysis tool that allows us to count the frequencies all triadic configurations in the network. We show that in tournaments of the same size, $t_{\text {tri }}$ and the linearity index $h$ both describe the degree to which triadic relations are transitive. Discrepancies between measures of linearity and triangle transitivity can arise when null dyads occur, with the linearity metric, $h^{\prime}$, becoming increasingly conservative with the proportion of null dyads. This is because the imputation procedure used to calculate linearity of incomplete networks $\left(h^{\prime}\right)$ creates triangles that do not exist in the observed data set, thus obscuring the actual triangle relationships that occurred. This effect is magnified with increasing group size because large matrices are more likely to be sparse. Sparse networks are ubiquitous: in our sample, $87 \%$ of dominance networks had at least one null dyad, and on average $26 \%$ of dyads were null. Of the 43 of 101 data sets in which $t_{\text {tri }}$ equalled 1 , only four were complete tournaments that could be considered unequivocally linear. We determined that 15 dominance hierarchies that had significantly high levels of triangle transitivity would not be considered significantly linear using the $h^{\prime}$ criterion (Figure 5). We argue that dominance relations in these groups are nonrandomly transitive. The decrease in $h^{\prime}$ associated with increasing group size is therefore largely explained by two factors: the average value of $h$ in randomized networks decreases with increasing group size, and large groups tend to contain more null dyads. In effect, social dynamics at the level of triads seems relatively unaffected by overall group size, such that triangle transitivity often remains high even in large groups. Thus, our findings emphasize even more strongly than heretofore appreciated the striking orderliness of most animal societies.

In the current study, we have scaled the triangle transitivity index relative to the null expected value. This scaling procedure presents some advantages and drawbacks. The major advantage of the $t_{\text {tri }}$ index is that it is scaled relative to a value that is constant regardless of network size or sparseness. The expected value of $t_{\text {tri }}$ is always 0 , and the maximum value is 1 , in all directed networks. Negative values of the $t_{\text {tri }}$ index are possible, but the exact minimum value, which will vary with network size and sparseness, is unknown. In the current study, we simply scaled $t_{\text {tri }}$ so as to be positive when triangles were more frequently transitive than average, and we scaled $t_{\text {tri }}$ to be negative when triangles were more frequently cyclic than the random expectation. The effect of network size and density on the bounds of indices of network structure is a general problem in network theory (Butts, 2006; Faust, 2010), and further work is needed to define precisely the bounds of triangle transitivity in sparse networks.

Our approach complements, but does not replace, other approaches to understanding the structure of dominance hierarchies. For example, we followed convention and assigned dominant-subordinate relations based on which individual won more than $50 \%$ of encounters, but this is most likely an oversimplification. Methods such as the directional consistency index (van Hooff \& Wensing 1987), a measure of how consistently one individual wins against another individual, may be useful for investigating the stability of dyadic relations. Moreover, there are other important dynamics of dominance relations that are not captured by the triangle transitivity metric. For example, even when all triangles are transitive $\left(P_{t}=t_{\text {tri }}=1\right)$, longer-range cycles (e.g. A > B > C > D > A) could occur, and these individuals cannot be arranged in orderly dominance ranks. Nevertheless, we focus on triads because it is the smallest unit of transitivity, and because the analysis of triads has been well established ( Holland and Leinhardt, 1976; Faust, 2007) and it offers ready comparison with existing measures of linearity. Other, more comprehensive network methods to analyse dominance data may be appropriate for some questions (Iverson \& Sade 1990). One such example is the dominance-directed tree method for generating graphical representations of nonlinear hierarchies (Izar et al. 2006). Similarly, more sophisticated measures of social structure that incorporate temporal and spatial dynamics are possible (Whitehead, 1997; Hemelrijk, 2000). Such dynamics are also central to network theory as well as studies of animal dominance. 
A major question remains: why do some pairs establish clear dominance relations while other pairs fail to interact? If patterns of noninteractions are not random, then this could bias the formation of transitive versus cyclic triangles. For example, if two individuals can infer their relative dominance status without interactions (e.g. via long-distance social signals), then individuals may avoid interacting with others when they are more likely to become entangled in an irresolvable cyclic triad. Third-party effects such as eavesdropping (Earley and Dugatkin, 2002; Mennill and Ratcliffe, 2004) can also affect whether certain individuals interact aggressively or avoid each other. Alternatively, some dyadic interactions may not be worth the cost of aggression, for example, if two similarly low-ranked individuals gain little benefit from outranking each other. With third-party effects and high-stakes contests, some individuals may avoid forming transitive triangles because the probable outcome of the contest is inferred through previous observations. Spatial segregation or other mechanisms that make it impossible for two individuals to interact (called structural zeros in de Vries 1995) would also affect which triads are closed, but whether this would bias triangle transitivity is not immediately clear. Theoretical models of the ecological and evolutionary processes that lead to particular patterns of 'triad closure' (Rapoport, 1953; Kossinets and Watts, 2006) will be important avenues of research to understand how highly transitive social dominance networks arise. Regardless of what mechanisms mediate competitive interactions, we suggest that patterns of noninteractions are critical to understanding social organization.

Social dominance is a dynamic process, and a network approach provides an opportunity to explore how social structure changes across time (Kossinets \& Watts 2006). Early sequences of dominance interactions can have profound effects on later interactions, as well as the global structure of the dominance network (e.g. winner and loser effects: Landau, 1951b; Dugatkin, 1997). For example, Chase (1982b) showed that in chickens, the potential levels of transitivity were largely determined by the early sequences of triadic interactions (i.e. when two out of the three dyads in a triad had established dominance relations). That is, two-edge configurations that ensured transitivity (i.e. 'Double-dominant' and 'Double-subordinate' in Figure 1) were more common than the only two-edge configuration that could potentially lead to cycles (i.e. 'Pass-along' in Figure 1). This imbalance between transitive and cyclic precursors at the two-edge stage formed the basis of the sequential development model of hierarchy formation (Chase, 1982b, 1985; Chase and Rohwer, 1987). The triad census provides a way to explore these dynamics efficiently on the level of the entire network through time. Because social interactions can often be observed in real time, sequential analyses of network structure should be a promising approach to connect these important lower-level processes (Faust 2007) to the patterns of hierarchical organization in dominance relations.

In general, the study of dominance hierarchies fits into a broad context of structure and transitivity in networks, a topic that unites physics, sociology, biology and other disciplines (Rapoport, 1953; Watts and Strogatz, 1998; Milo et al., 2002; Barabási, 2009). Network analyses play an important and increasing role in exploring the structure of animal societies (Krause et al., 2007; Wey et al., 2007; Sih et al., 2009). We anticipate that observations of nonhuman animal social systems in natural and experimental contexts will have much to contribute to the general understanding of the processes that lead to orderly social structures in nature.

Acknowledgments - We thank Andrew Edelman, Shannon Albeke, Steven Prager and Bryan Shader for fruitful discussions and feedback on ideas, and Trevor Price and two anonymous referees for constructive comments this manuscript. This study was funded by National Science Foundation grant DEB-0918736 to D.B.M. D.S. was supported by Chicago Fellows postdoctoral fellowship at the University of Chicago during the preparation of this manuscript.

\section{References}

Allesina, S. \& Levine, J. M. 2011. A competitive network theory of species diversity. Proceedings of the National Academy of Sciences U.S.A., 108, 5638-5642.

Appleby, M. C. 1983. The probability of linearity in hierarchies. Animal Behaviour, 31, 600e608.

Barabási, A.-L. 2009. Scale-free networks: A decade and beyond. Science, 325, 412-413.

Butts, C. T. 2006. Exact bounds for degree centralization. Social Networks, 28, 283-296.

Cant, M. A., English, S., Reeve, H. K. \& Field, J. 2006. Escalated conflict in a social hierarchy. Proceedings of the Royal Society B, 273, 2977-2984.

Chase, I. D. 1982a. Dynamics of hierarchy formation: the sequential development of dominance relationships. Behaviour, 80, 218-240.

Chase, I. D. 1982b. Behavioral sequences during dominance hierarchy formation in chickens. Science, 216, 439-440.

Chase, I. D. 1985. The sequential analysis of aggressive acts during hierarchy formation: an application of the 'jigsaw puzzle' approach. Animal Behaviour, 33, 86-100.

Chase, I. D. \& Rohwer, S. 1987. Two methods for quantifying the development of dominance hierarchies in large groups with applications to Harris' sparrows. Animal Behaviour, 35, 1113-1128.

Csárdi, G. \& Nepusz, T. 2006. The igraph software package for complex network research. InterJournal Complex Systems, 1695, http:/ /igraph.sf.net

Dawkins, R. 1976. Hierarchical organisation: a candidate principle for ethology. In: Growing Points in Ethology (Ed. by P. Bateson \& R. Hinde), pp. 7-54. Cambridge: Cambridge University Press.

Drews, C. 1993. The concept and definition of dominance in animal behaviour. Behaviour, 125, 283-313.

van Doorn, G. S., Hengeveld, G. M. \& Weissing, F. J. 2003. The evolution of social dominance I: Two-player models. Behaviour, 140, 1305-1332.

Dugatkin, L. 1997. Winner and loser effects and the structure of dominance hierarchies. Behavioral Ecology, 8, 583-587.

Earley, R. L. \& Dugatkin, L. A. 2002. Eavesdropping on visual cues in green swordtail (Xiphophorus helleri) fights: A case for networking. Proceedings of the Royal Society of London, Series B, 269, 943-952.

Ellis, L. 1995. Dominance and reproductive success among nonhuman animals: A cross-species comparison. Ethology and Sociobiology, 16, 257-333.

Faust, K. 2007. Very local structure in social networks. Sociological Methodology, 37, 209-256.

Faust, K. 2008. Triadic configurations in limited choice sociometric networks: Empirical and theoretical results. Social Networks, 30, 273e282.

Faust, K. 2010. A puzzle concerning triads in social networks: graph constraints and the triad census. Social Networks, 32, 221-233.

Frank, O. \& Harary, F. 1982. Cluster inference by using transitivity indices in empirical graphs. Journal of the American Statistical Association, 77, 835-840.

Handcock, M., Hunter, D., Butts, C., Goodreau, S. \& Morris, M. 2003. Statnet: Software Tools for the Statistical Modeling of Network Data. http://statnetproject.org

Harary, F. \& Moser, L. 1966. The theory of round robin tournaments. American Mathematical Monthly, 73, 231-246.

Hemelrijk, C. K. 2000. Towards the integration of social domi- 
nance and spatial structure. Animal Behaviour, 59, 1035-1048.

Holland, P. W. \& Leinhardt, S. 1970. A method for detecting structure in sociometric data. American Journal of Sociology, 76, 492-513.

Holland, P. W. \& Leinhardt, S. 1976. Local structure in social networks. Sociological Methodology, 7, 1e45.

van Hooff, J. A. R. A. M. \& Wensing, J. A. B. 1987. Dominance and its behavioral measures in a captive wolf pack. In: Man and Wolf: Advances, Issues, and Problems in Captive Wolf Research (Ed. by H. Frank), pp. 219e252. Dordrecht: Junk.

Iverson, G. \& Sade, D. 1990. Statistical issues in the analysis of dominance hierarchies in animal societies. Journal of Quantitative Anthropology, 2, 61e83.

Izar, P., Ferreira, R. G. \& Sato, T. 2006. Describing the organization of dominance relationships by dominance-directed tree method. American Journal of Primatology, 68, 189e207.

Karlberg, M. 1999. Testing transitivity in digraphs. Sociological Methodology, 29, 225-251.

Kendall, M. \& Babington Smith, B. 1940. On the method of paired comparisons. Biometrika, 31, 324-.

Klass, K. \& Cords, M. 2011. Effect of unknown relationships on linearity, steepness and rank ordering of dominance hierarchies: simulation studies based on data from wild monkeys. Behavioural Processes, 88, 168-176.

Kossinets, G. \& Watts, D. J. 2006. Empirical analysis of an evolving social network. Science, 311, 88-90.

Krause, J., Croft, D. \& James, R. 2007. Social network theory in the behavioural sciences: potential applications. Behavioral Ecology and Sociobiology, 62, 15-27.

Landau, H. G. 1951a. On dominance relations and the structure of animal societies: I. Effect of inherent characteristics. Bulletin of Mathematical Biology, 13, 1-19.

Landau, H. G. 1951b. On dominance relations and the structure of animal societies: II. Some effects of possible social factors. Bulletin of Mathematical Biology, 13, 245-262.

Mennill, D. \& Ratcliffe, L. 2004. Do male black-capped chickadees eavesdrop on song contests? A multi-speaker playback experiment. Behaviour, 141, 125-139.

Milo, R., Shen-Orr, S., Itzkovitz, S., Kashtan, N., Chklovskil, D. \& Alon, U. 2002. Network motifs: simple building blocks of complex networks. Science, 298, 824-827.

Nakagawa, S. \& Freckleton, R. P. 2008. Missing inaction: the dangers of ignoring missing data. Trends in Ecology \& Evolution, 23, 592-596.

R Development Core Team 2009. R: A Language and Environment for Statistical Computing. Vienna: R Foundation for Statistical Computing. http:/ / www.Rproject.org

Rapoport, A. 1953. Spread of information through a population with sociostructural bias: I. Assumption of transitivity. Bulletin of Mathematical Biology, 15, 523-533.

Schjelderup-Ebbe, T. 1922. Beitrlge zur Sozialpsychologie des Haushuhns. Zeitschrift für Psychologie, 88, 225-252.

Sih, A., Hanser, S. F. \& Mchugh, K. A. 2009. Social network theory: new insights and issues for behavioral ecologists. Behavioral Ecology and Sociobiology, 63, 975-988.

de Vries, H. 1995. An improved test of linearity in dominance hierarchies containing unknown or tied relationships. Animal Behaviour, 50, 1375-1389.

Wasserman, S. \& Faust, K. 1994. Social Network Analysis: Methods and Applications. Cambridge: Cambridge University Press.

Watts, D. \& Strogatz, S. 1998. Collective dynamics of 'smallworld'networks. Nature, 393, 440-442.

Wey, T., Blumstein, D., Shen, W. \& Jordán, F. 2007. Social network analysis of animal behaviour: A promising tool for the study of sociality. Animal Behaviour, 74, 333-344.

Whitehead, H. 1997. Analysing animal social structure. Animal Behaviour, 53, 1053-1067
Appendix 1.

\section{Deriving the Expected Value of $P_{t}$ in Random Networks}

In this study, we use a simulation approach to generate the distribution of $P_{t}$ under the assumption of random dominance relations. The distributions of expected frequencies of triadic configurations for random graphs conditioned on the number of mutual, asymmetrical and null dyads (termed U |MAN distribution) was derived by Holland \& Leinhardt (1976). We can use this to simply derive the average expected value of $P_{t}$ : the proportion of triangles that are transitive. Following Holland \& Leinhardt (1976),

$$
\begin{aligned}
& E_{(030 \mathrm{C})}=1 / 4 \frac{A^{(3)}}{k^{(3)}} \\
& E_{(030 \mathrm{~T})}=3 / 4 \frac{A^{(3)}}{k^{(3)}}
\end{aligned}
$$

where $E_{(030 \mathrm{~T})}$ and $E_{(030 \mathrm{C})}$ are expected frequencies of transitive and cyclic triangles, $A$ is the number of asymmetrical edges in the network, and $k$ is the number of dyads in network $g$. The notation $z^{(3)}$ stands for $z \times(z-1) \times(z-2)$. The expected value of $P_{t}$ in random networks, $E\left(P_{t}\right)$ is then:

$$
E\left(P_{t}\right)=\frac{E_{(030 \mathrm{~T})}}{E_{(030 \mathrm{~T})}+E_{(030 \mathrm{C})}}=\frac{3}{4}
$$

Therefore, the expected average proportion of transitive triangles in a directed network is 0.75 . This is confirmed independently in the main text using simulations and by a heuristic argument for the six transitive triads among the eight possible ways of adding three edges to a given triad (see Results and Figure 2).

\section{Appendix 2.}

\section{Calculating $P_{t}$ in Networks with Mutual Dyads}

In the analysis presented in the main text, we excluded mutual dyads (dyads in which both individuals win the same number of contests) because these dyads are rare and have only slight effects on measurements of triad transitivity. However, our approach is easily extended to include dominance networks with mutual dyads.

We first consider the seven different triangle configurations that are possible in networks with mutual, asymmetrical and null dyads (indicated in black in Figure A1). Following Holland \& Leinhardt (1976), each triad configuration is labelled with a three-number code representing the number of mutual, asymmetric and null dyads (Figure A1). When applicable, a fourth character is used to denote distinct configurations that share the same frequencies of dyad types $\left({ }^{\prime} \mathrm{D}^{\prime}=\right.$ down, ' $\mathrm{U}^{\prime}=$ up, ' $\mathrm{C}^{\prime}=$ cycle, ' $\mathrm{T}$ ' = transitive). For example, a transitive triad has no mutual dyads, three asymmetrical dyads and no null dyads, so it is labelled as '030T'. This is distinguished from a cyclic triad that also contains only three asymmetrical dyads, labelled '030C'. This labelling scheme is referred to as MAN labelling (for mutual, aymmetric and null dyads: Wasserman \& Faust 1994). 


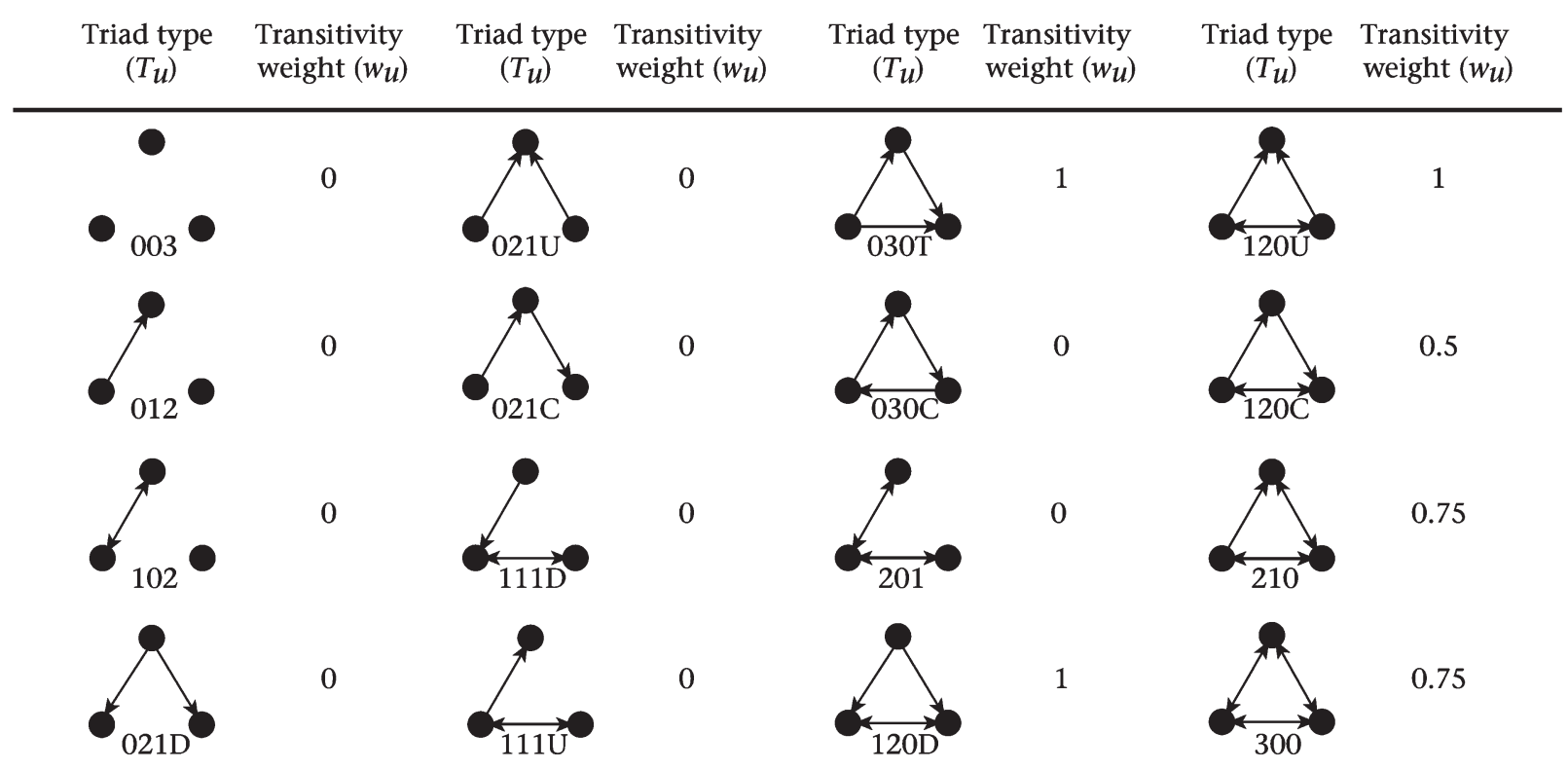

Figure A1. Full triad census, showing all 16 triad configurations possible in a directed network. Each configuration is named using the MAN labelling scheme (see text; Holland \& Leinhardt 1976). Seven triangle configurations, in which all three nodes are connected by either asymmetric or mutual edges, are shown in black. Weighting factor $\left(w_{u}\right)$ for each of the seven triangle configuration types is based on the probability that the triangle is transitive, with the assumption that each individual in a mutual dyad has equal probability of being dominant. Using these weighting factors, we can calculate the proportion of transitive triangles $\left(P_{t}\right)$ in any directed network (equation A1).

Triads that contain mutual dyads are weighted according to the probability that the configuration is transitive if the mutual dyad becomes a dominant-subordinate relation (Holland and Leinhardt, 1976; Wasserman and Faust, 1994). This is akin to the treatment of mutual dyads in measuring linearity (Appleby, 1983; de Vries, 1995): both individuals are considered to have equal chance of being dominant. For example, triad 120C is weighted by a value of 0.5 ; the triad may be transitive or intransitive, depending on which member of the mutual dyad dominates, with both scenarios equally probable. Furthermore, 120D and 120U are transitive no matter which individual in the mutual dyad is dominant, so this is weighted by a value of 1 . Triad types 210 and 300 are weighted by 0.75 using the same logic. The weighting factors $\left(w_{u}\right)$ of all triad types are presented in Figure A1. Note that $P_{t}$ cannot be measured if no triangle triads occur.

Applying these transitivity weights to each triad configuration we can now calculate $P_{t}$ for networks with mutual dyads.

$$
P_{t}=\frac{\sum_{u} w_{u} T_{u}}{N_{\text {triangles }}}
$$

where $T_{u}$ is the frequency of triad type $u$, and $w_{u}$ is the weighting factor for transitivity for triad $u$. $N_{\text {triangles }}$ is the total number of triangles in the network. The $t_{\text {tri }}$ index can be calculated by taking this $P_{t}$ value and applying equation (6). The $P_{t}$ values calculated for empirical networks including or excluding mutual dyads were highly correlated $\left(r_{99}=0.92\right.$, $N=101, P<0.001)$, and the mean difference in value was 0.006 . Therefore, excluding the mutual dyads, at least in this sample of 101 data sets, did not change our results. However, in other networks where mutual dyads are more common, this equation (A1) may be a more appropriate method of calculating $P_{t}$.

The statistical procedure for generating a $P$ value for the triangle transitivity of an empirical network is the same as outlined in the main text. For each empirical network, we generate 1000 dyad census-conditioned random graphs and calculate $t_{\text {tri }}$ for each. The one-tailed $P$ value is the number of times the $t_{\text {tri }}$ value of the random network is greater than the $t_{\text {tri }}$ value of the empirical network. In the Supplementary Material, we provide the codes for conducting this procedure in $\mathrm{R}$. 


\section{Supplementary Material}

Calculation and Statistical Test of Triangle Transitivity

Here, we provide the codes to calculate $P_{t}$ and $t_{\text {tri }}$ and to conduct a statistical test of triangle transitivity of an empirical network using the package 'statnet' (Handcock et al. 2003) in the R programming environment (R Development Core Team 2009). The following codes calculate $P_{t}$ and $t_{\text {tri }}$ in networks using the methods described in Appendix 2.

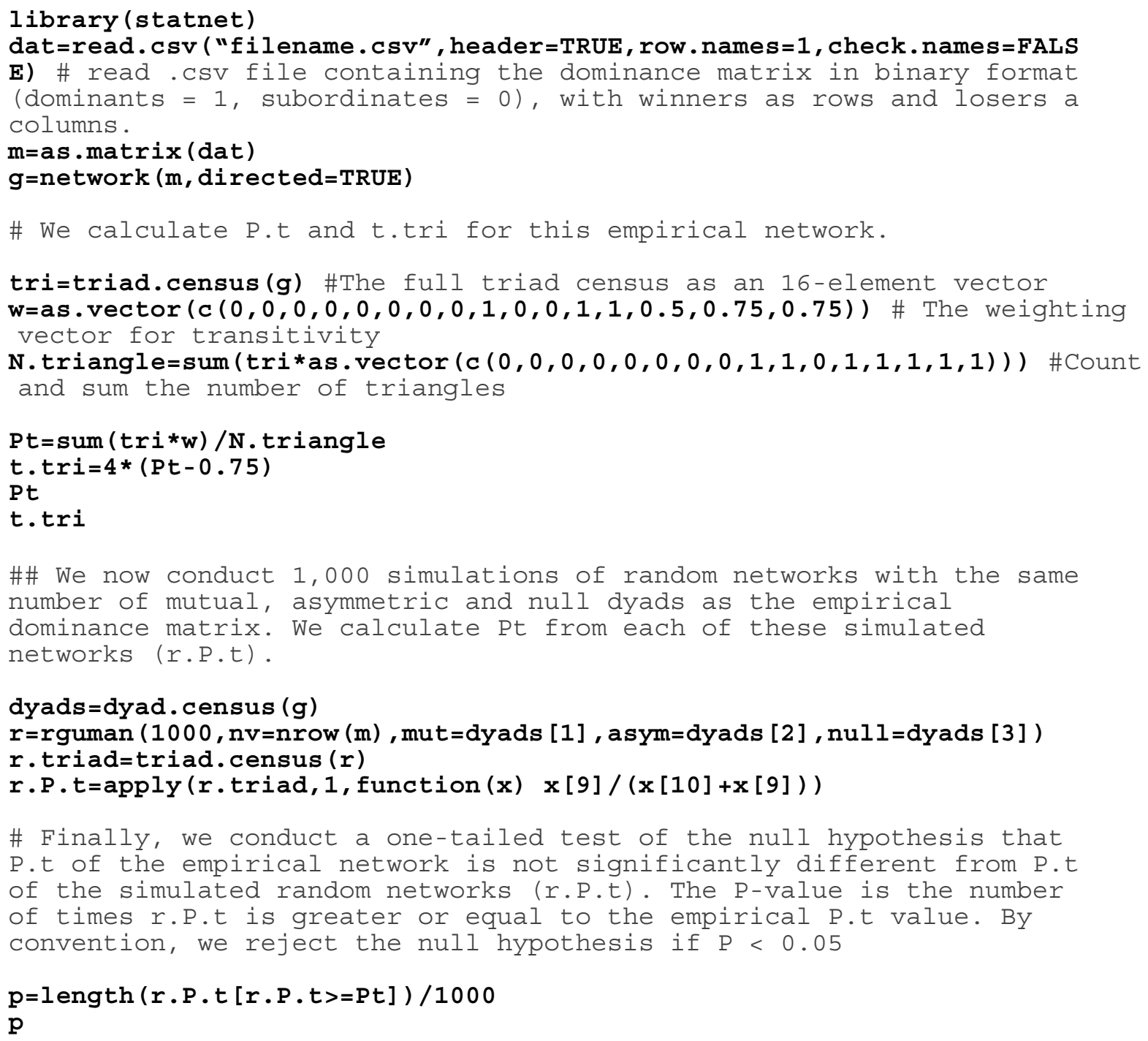




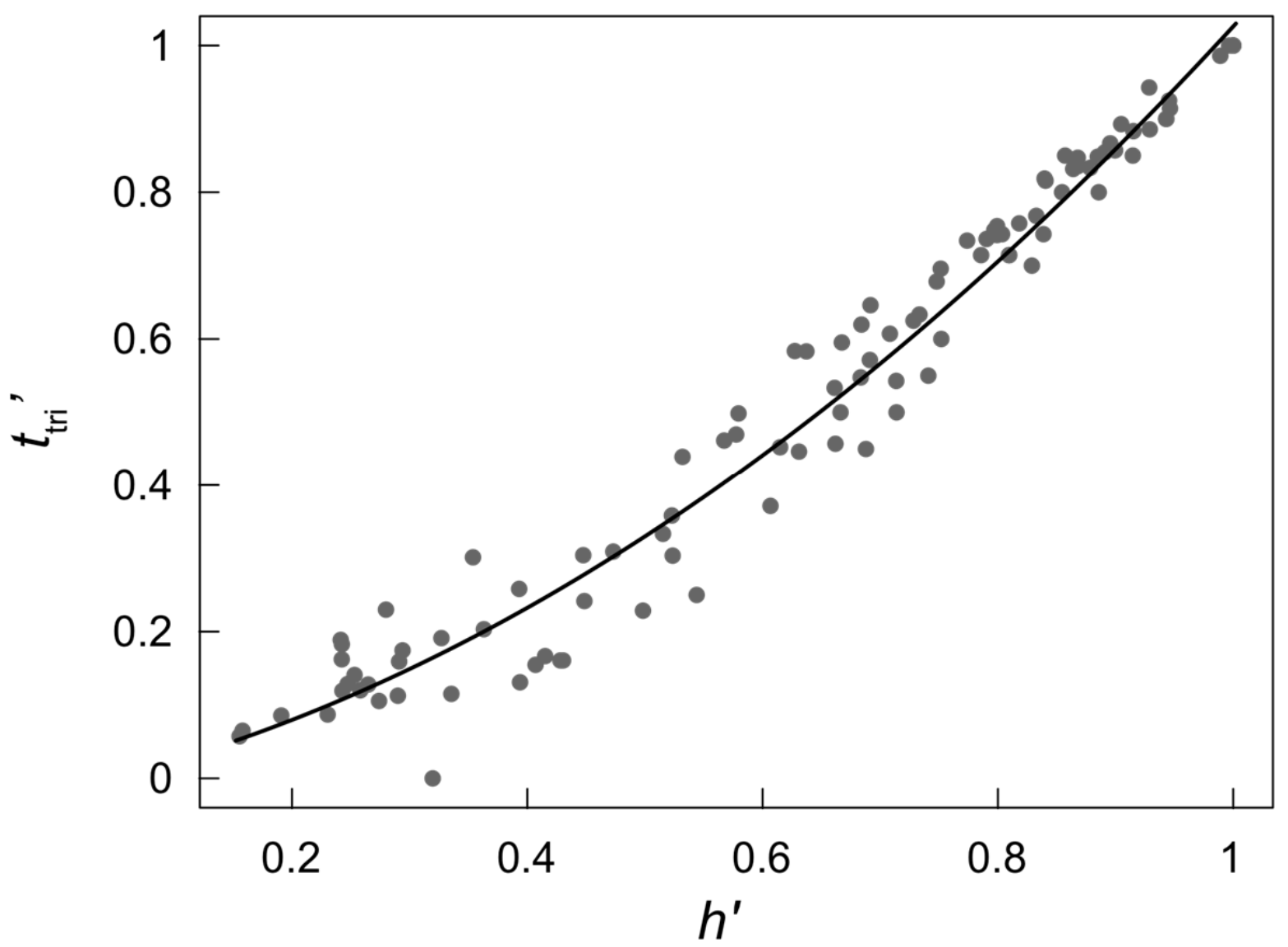

Figure S1. Relationship between $t_{\text {tri }}{ }^{\prime}$ and $h^{\prime}$ measured using simulations to fill in null dyads from empirical matrices. The line is a polynomial regression line $\left(Y=-0.02+0.35 \times X+0.70 \times X^{2} ; F_{2,98}=1491, R_{\text {adj }}^{2}=0.97, P\right.$ $<0.001)$. The two values are closely related but not directly proportional because the exact relationship between the two indices depends on the size of the network (i.e. the number of individuals). 

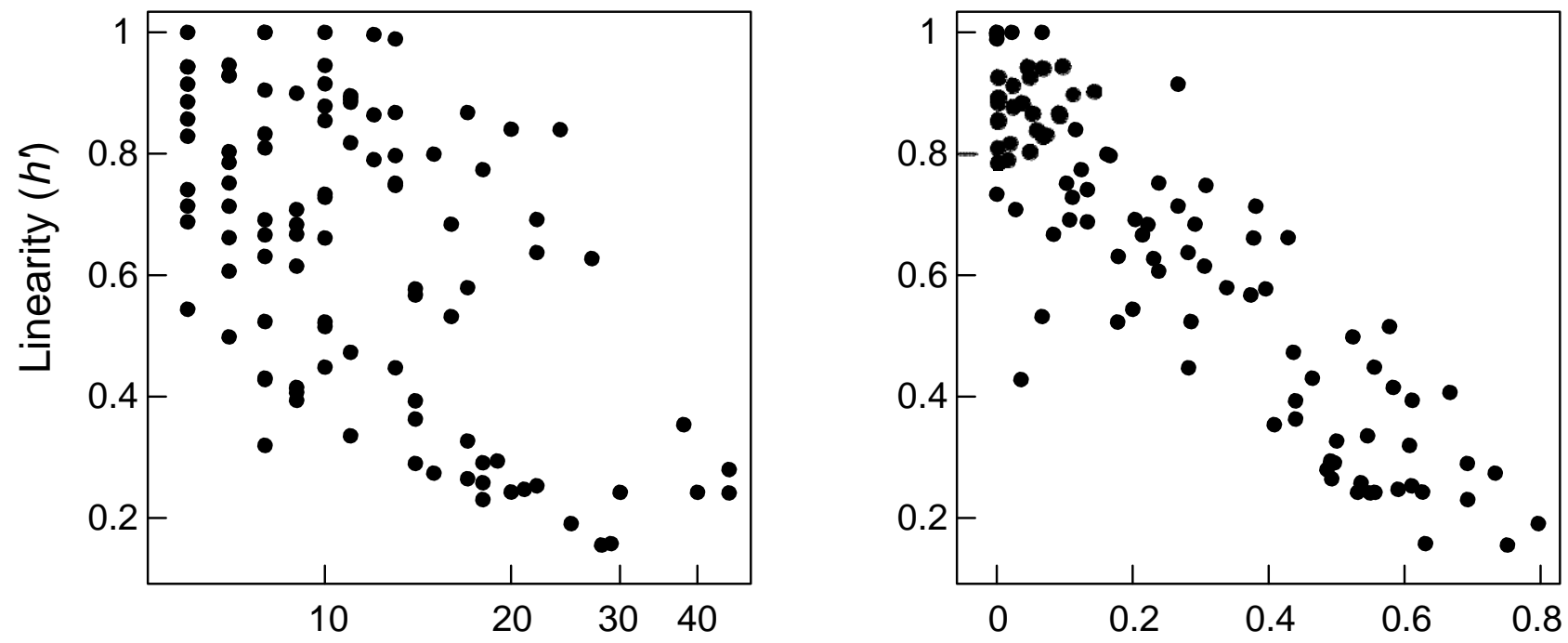

(c)

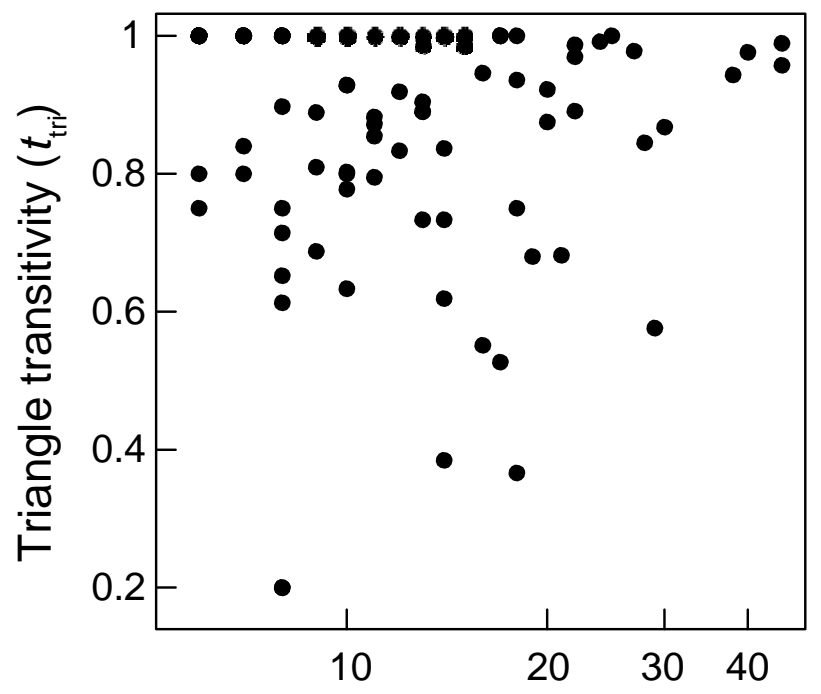

Number of individuals (log)

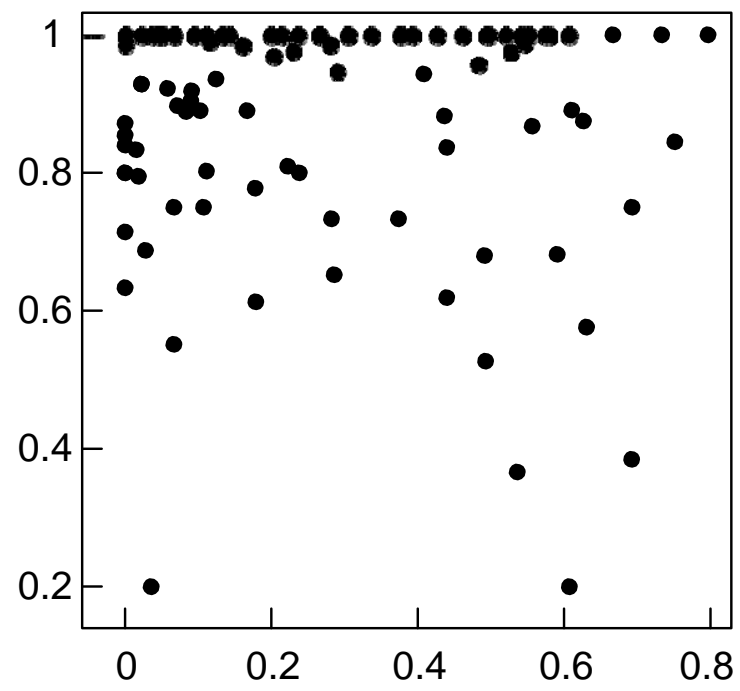

Network sparseness

Figure S2. Measures of linearity and triangle transitivity in relation to network size (number of individuals) and network sparseness (proportion of dyads with unknown dominance relationship). (a, b) The linearity measure, $h^{\prime}$, decreases with increasing numbers of individuals and proportions of dyads unknown. This bias is created by the process of filling in null dyads with random dominance relationships. (c, d) Triangle transitivity, $t_{\text {tri, }}$ is not affected by the number of individuals or the proportion of dyads known. 


\section{Table S1}

Empirical dominance matrices used for analysis of $t_{\mathrm{tr}}, t_{\mathrm{tri}}{ }^{\prime}$ and $h^{\prime}$

\begin{tabular}{|c|c|c|c|c|c|c|c|c|}
\hline \multirow[t]{2}{*}{ Source } & \multicolumn{2}{|c|}{ Network } & \multirow[t]{2}{*}{$P_{t}$} & \multirow[t]{2}{*}{$t_{\text {tri }}$} & \multirow[t]{2}{*}{$t_{\mathrm{tri}}{ }^{\prime}$} & \multirow[t]{2}{*}{$h^{\prime}$} & \multicolumn{2}{|c|}{$P$ value } \\
\hline & Size & Sparseness & & & & & $h^{\prime}$ & $t_{\mathrm{tri}}$ \\
\hline Allee \& Dickinson 1954, Table 3 & 10 & 0.378 & 1.000 & 1.000 & 0.533 & 0.661 & 0.012 & 0.000 \\
\hline Appleby 1983, Figure 1 & 7 & 0.381 & 1.000 & 1.000 & 0.543 & 0.714 & 0.098 & 0.152 \\
\hline Archie et al. 2006, Figure 2 (GB) & 11 & 0.545 & 1.000 & 1.000 & 0.115 & 0.335 & 0.311 & 0.033 \\
\hline Archie et al. 2006, Figure $2(A)$ & 8 & 0.214 & 1.000 & 1.000 & 0.500 & 0.666 & 0.094 & 0.005 \\
\hline Archie et al. 2006, Figure 2 (AA) & 10 & 0.556 & 1.000 & 1.000 & 0.242 & 0.449 & 0.174 & 0.079 \\
\hline Archie et al. 2006, Figure 2 (CB) & 6 & 0.067 & 1.000 & 1.000 & 1.000 & 1.000 & 0.021 & 0.033 \\
\hline Archie et al. 2006, Figure 2 (FB) & 6 & 0.133 & 1.000 & 1.000 & 0.450 & 0.688 & 0.258 & 0.057 \\
\hline Archie et al. 2006, Figure 2 (JA) & 7 & 0.429 & 1.000 & 1.000 & 0.457 & 0.662 & 0.168 & 0.213 \\
\hline Archie et al. 2006, Figure 2 (OA) & 9 & 0.611 & 1.000 & 1.000 & 0.131 & 0.394 & 0.354 & 0.350 \\
\hline Archie et al. 2006, Figure 2 (PC) & 9 & 0.667 & 1.000 & 1.000 & 0.155 & 0.407 & 0.324 & 0.484 \\
\hline Archie et al. 2006, Figure 2 (SI) & 6 & 0.067 & 1.000 & 1.000 & 0.900 & 0.943 & 0.036 & 0.046 \\
\hline Barrette \& Vandal 1986, Table 2 & 20 & 0.058 & 0.981 & 0.922 & 0.816 & 0.840 & 0.000 & 0.000 \\
\hline Berman et al. 2004, Appendix A & 22 & 0.203 & 0.992 & 0.970 & 0.646 & 0.692 & 0.000 & 0.000 \\
\hline Berman et al. 2004, Appendix B & 22 & 0.281 & 0.997 & 0.987 & 0.583 & 0.637 & 0.000 & 0.000 \\
\hline Berman et al. 2004, Appendix C & 18 & 0.124 & 0.984 & 0.936 & 0.734 & 0.774 & 0.000 & 0.000 \\
\hline Bonanni et al. 2007, Table 1 & 13 & 0.282 & 0.933 & 0.733 & 0.304 & 0.448 & 0.025 & 0.000 \\
\hline Bonanni et al. 2007, Table 2 & 14 & 0.374 & 0.990 & 0.960 & 0.462 & 0.567 & 0.000 & 0.000 \\
\hline Cafazzo et al. 2010, Table 5 & 27 & 0.231 & 0.994 & 0.978 & 0.584 & 0.627 & 0.000 & 0.000 \\
\hline Cheney 1977, Table 1a & 12 & 0.000 & 1.000 & 1.000 & 1.000 & 0.997 & 0.000 & 0.000 \\
\hline Cheney 1977, Table $1 \mathrm{~b}$ & 12 & 0.015 & 0.958 & 0.833 & 0.736 & 0.790 & 0.000 & 0.000 \\
\hline Clutton-Brock et al. 1976, Table 3 & 17 & 0.051 & 1.000 & 1.000 & 0.847 & 0.868 & 0.000 & 0.000 \\
\hline Collias \& Taber 1951, Table 3 & 9 & 0.583 & 1.000 & 1.000 & 0.167 & 0.415 & 0.300 & 0.260 \\
\hline Collias Taber 1951, Table 4 & 8 & 0.464 & 1.000 & 1.000 & 0.161 & 0.430 & 0.325 & 0.097 \\
\hline Collias Taber 1951, Table 5 & 14 & 0.440 & 0.905 & 0.619 & 0.203 & 0.363 & 0.071 & 0.002 \\
\hline
\end{tabular}




\begin{tabular}{|c|c|c|c|c|c|c|c|c|}
\hline \multirow[t]{2}{*}{ Source } & \multicolumn{2}{|c|}{ Network } & \multirow[t]{2}{*}{$P_{t}$} & \multirow[t]{2}{*}{$t_{\text {tri }}$} & \multirow[t]{2}{*}{$t_{\mathrm{tri}}{ }^{\prime}$} & \multirow[t]{2}{*}{$h^{\prime}$} & \multicolumn{2}{|c|}{$P$ value } \\
\hline & Size & Sparseness & & & & & $h^{\prime}$ & $t_{\text {tri }}$ \\
\hline Côté 2000, Table A1 & 38 & 0.408 & 0.986 & 0.943 & 0.301 & 0.354 & 0.000 & 0.000 \\
\hline Côté 2000, Table A2 & 40 & 0.531 & 0.994 & 0.976 & 0.183 & 0.242 & 0.000 & 0.000 \\
\hline Côté 2000, Table A3 & 45 & 0.549 & 0.997 & 0.989 & 0.189 & 0.241 & 0.000 & 0.000 \\
\hline Côté 2000, Table A4 & 45 & 0.486 & 0.989 & 0.957 & 0.230 & 0.280 & 0.000 & 0.000 \\
\hline Farentinos 1972, Table 1A & 6 & 0.267 & 1.000 & 1.000 & 0.500 & 0.714 & 0.209 & 0.157 \\
\hline Farentinos 1972, Table 1B & 8 & 0.286 & 0.913 & 0.652 & 0.304 & 0.524 & 0.172 & 0.045 \\
\hline Farentinos 1972, Table $1 \mathrm{C}$ & 8 & 0.607 & 0.800 & 0.200 & 0.000 & 0.320 & 0.519 & 0.641 \\
\hline Farentinos 1972, Table 1D & 11 & 0.436 & 0.971 & 0.882 & 0.309 & 0.473 & 0.067 & 0.007 \\
\hline Farentinos 1972, Table 1E & 6 & 0.000 & 0.950 & 0.800 & 0.800 & 0.886 & 0.051 & 0.058 \\
\hline Fournier \& Festa-Bianchet 1995, Figure 3 & 30 & 0.556 & 0.967 & 0.868 & 0.162 & 0.242 & 0.000 & 0.000 \\
\hline Fournier 1995 \& Festa-Bianchet, Figure 2 & 21 & 0.590 & 0.920 & 0.682 & 0.129 & 0.247 & 0.048 & 0.000 \\
\hline Frank 1986, Table 1 & 25 & 0.797 & 1.000 & 1.000 & 0.086 & 0.191 & 0.072 & 0.008 \\
\hline Hartzler 1970, Table 1 & 7 & 0.095 & 1.000 & 1.000 & 0.914 & 0.946 & 0.007 & 0.005 \\
\hline Hass \& Jenni 1991, Table 1 & 15 & 0.733 & 1.000 & 1.000 & 0.105 & 0.274 & 0.204 & 0.123 \\
\hline Hass \& Jenni 1991, Table 2 & 18 & 0.497 & 1.000 & 1.000 & 0.159 & 0.291 & 0.063 & 0.000 \\
\hline Hass \& Jenni 1991, Table 3 & 17 & 0.500 & 1.000 & 1.000 & 0.191 & 0.327 & 0.041 & 0.000 \\
\hline Heitor \& Vicente 2010, Table 2 & 6 & 0.000 & 1.000 & 1.000 & 0.850 & 0.857 & 0.049 & 0.021 \\
\hline Heitor et al. 2006, Table 3 & 11 & 0.000 & 0.964 & 0.855 & 0.855 & 0.891 & 0.000 & 0.000 \\
\hline Hewitt et al. 2009, Figure A1 (SH1995) & 14 & 0.440 & 0.959 & 0.837 & 0.258 & 0.393 & 0.038 & 0.000 \\
\hline Hewitt et al. 2009, Figure A1 (P2004) & 7 & 0.000 & 1.000 & 1.000 & 0.943 & 0.929 & 0.005 & 0.000 \\
\hline Hewitt et al. 2009, Figure A1 (PO2004) & 7 & 0.238 & 0.950 & 0.800 & 0.371 & 0.607 & 0.206 & 0.026 \\
\hline Hewitt et al. 2009, Figure A1 (P2005) & 9 & 0.222 & 0.952 & 0.810 & 0.548 & 0.683 & 0.018 & 0.003 \\
\hline Hirotani 1994, Table 1 & 13 & 0.090 & 0.976 & 0.904 & 0.836 & 0.868 & 0.000 & 0.000 \\
\hline Isbell \& Pruet 1998, Figure $1 \mathrm{~A}$ & 9 & 0.306 & 1.000 & 1.000 & 0.452 & 0.615 & 0.057 & 0.001 \\
\hline Isbell \& Pruet 1998, Figure 1B & 17 & 0.493 & 0.882 & 0.527 & 0.128 & 0.265 & 0.111 & 0.002 \\
\hline Izar et al. 2006, Table 2 & 17 & 0.338 & 1.000 & 1.000 & 0.499 & 0.580 & 0.000 & 0.000 \\
\hline Koenig et al. 2004, Table $1 b$ & 7 & 0.048 & 1.000 & 1.000 & 0.743 & 0.804 & 0.024 & 0.007 \\
\hline
\end{tabular}




\begin{tabular}{|c|c|c|c|c|c|c|c|c|}
\hline \multirow[t]{2}{*}{ Source } & \multicolumn{2}{|c|}{ Network } & \multirow[t]{2}{*}{$P_{t}$} & \multirow[t]{2}{*}{$t_{\text {tri }}$} & \multirow[t]{2}{*}{$t_{\text {tri }}{ }^{\prime}$} & \multirow[t]{2}{*}{$h^{\prime}$} & \multicolumn{2}{|c|}{$P$ value } \\
\hline & Size & Sparseness & & & & & $h^{\prime}$ & $t_{\text {tri }}$ \\
\hline Lahti et al. 1994, Table 1A & 6 & 0.067 & 0.938 & 0.750 & 0.700 & 0.829 & 0.084 & 0.077 \\
\hline Lahti et al. 1994, Table 1C & 8 & 0.071 & 0.974 & 0.897 & 0.768 & 0.833 & 0.002 & 0.002 \\
\hline Lu et al. 2008, Figure 1c(1) & 13 & 0.103 & 0.973 & 0.890 & 0.696 & 0.751 & 0.000 & 0.000 \\
\hline Lu et al. 2008, Figure 1c(2) & 13 & 0.167 & 0.973 & 0.890 & 0.748 & 0.797 & 0.000 & 0.000 \\
\hline McMahan \& Morris 1984, Table 1 & 10 & 0.178 & 0.944 & 0.778 & 0.358 & 0.523 & 0.073 & 0.000 \\
\hline Marler 1957, Table $1 b$ & 8 & 0.000 & 1.000 & 1.000 & 1.000 & 1.000 & 0.000 & 0.000 \\
\hline Masure \& Allee 1934, Table 1 & 13 & 0.000 & 0.997 & 0.986 & 0.986 & 0.989 & 0.000 & 0.000 \\
\hline Masure \& Allee 1934, Table 2 & 11 & 0.018 & 0.949 & 0.795 & 0.758 & 0.818 & 0.000 & 0.000 \\
\hline Masure \& Allee 1934, Table 3 & 7 & 0.000 & 0.960 & 0.840 & 0.714 & 0.786 & 0.033 & 0.006 \\
\hline Møller 1987, Table 1 & 13 & 0.308 & 1.000 & 1.000 & 0.678 & 0.748 & 0.000 & 0.000 \\
\hline Møller 1987, Table 2 & 10 & 0.044 & 1.000 & 1.000 & 0.925 & 0.946 & 0.000 & 0.000 \\
\hline Møller 1987, Table 3 & 14 & 0.396 & 1.000 & 1.000 & 0.470 & 0.578 & 0.001 & 0.000 \\
\hline Monnin \& Peeters 1999, Table 1 & 6 & 0.200 & 1.000 & 1.000 & 0.250 & 0.544 & 0.280 & 0.208 \\
\hline Murray 2007, Table 5 & 18 & 0.693 & 0.938 & 0.750 & 0.087 & 0.230 & 0.177 & 0.024 \\
\hline Nakano 1994, Table 1 & 11 & 0.036 & 1.000 & 1.000 & 0.848 & 0.885 & 0.000 & 0.000 \\
\hline Natoli \& De Vito 1991, Table 2 & 14 & 0.692 & 0.846 & 0.385 & 0.113 & 0.290 & 0.215 & 0.296 \\
\hline Owens \& Owens 1996, Figure 1 & 7 & 0.238 & 1.000 & 1.000 & 0.600 & 0.752 & 0.080 & 0.034 \\
\hline Paoli et al. 2006, Table 2 & 8 & 0.036 & 0.800 & 0.200 & 0.161 & 0.428 & 0.251 & 0.224 \\
\hline Paoli et al. 2006, Table 3 & 6 & 0.267 & 1.000 & 1.000 & 0.850 & 0.915 & 0.052 & 0.152 \\
\hline Parsons \& Baptista 1980, Table 4 & 8 & 0.000 & 0.929 & 0.714 & 0.714 & 0.810 & 0.006 & 0.004 \\
\hline Patterson 1977, Figure 5 & 18 & 0.536 & 0.842 & 0.366 & 0.120 & 0.258 & 0.092 & 0.038 \\
\hline Patterson 1977, Figure 6 & 28 & 0.751 & 0.961 & 0.845 & 0.057 & 0.155 & 0.116 & 0.001 \\
\hline Poisbleau et al. 2006, Figure 2a & 16 & 0.067 & 0.888 & 0.551 & 0.439 & 0.532 & 0.000 & 0.000 \\
\hline Poisbleau et al. 2006, Figure $2 b$ & 19 & 0.491 & 0.920 & 0.680 & 0.174 & 0.294 & 0.028 & 0.000 \\
\hline Richter et al. 2009, Table 1 & 9 & 0.028 & 0.922 & 0.688 & 0.607 & 0.708 & 0.005 & 0.001 \\
\hline Robbins 2008, Table 2 & 8 & 0.179 & 0.903 & 0.613 & 0.446 & 0.631 & 0.071 & 0.031 \\
\hline Rutberg 1986, Table 1 & 22 & 0.610 & 0.973 & 0.891 & 0.141 & 0.253 & 0.026 & 0.000 \\
\hline
\end{tabular}




\begin{tabular}{|c|c|c|c|c|c|c|c|c|}
\hline \multirow[t]{2}{*}{ Source } & \multicolumn{2}{|c|}{ Network } & \multirow[t]{2}{*}{$P_{t}$} & \multirow[t]{2}{*}{$t_{\text {tri }}$} & \multirow[t]{2}{*}{$t_{\mathrm{tri}}{ }^{\prime}$} & \multirow[t]{2}{*}{$h^{\prime}$} & \multicolumn{2}{|c|}{$P$ value } \\
\hline & Size & Sparseness & & & & & $h^{\prime}$ & $t_{\text {tri }}$ \\
\hline Rutberg 1986, Table 2 & 29 & 0.631 & 0.894 & 0.576 & 0.065 & 0.158 & 0.083 & 0.000 \\
\hline Satoh \& Ohkawara 2008, Table 2c & 8 & 0.143 & 1.000 & 1.000 & 0.893 & 0.905 & 0.001 & 0.000 \\
\hline Satoh \& Ohkawara 2008, Table 2d & 9 & 0.083 & 0.972 & 0.889 & 0.595 & 0.667 & 0.013 & 0.000 \\
\hline Satoh \& Ohkawara 2008, Table 2e & 10 & 0.578 & 1.000 & 1.000 & 0.333 & 0.515 & 0.076 & 0.110 \\
\hline Setchell \& Wickings 2005, Table 4 & 8 & 0.000 & 1.000 & 1.000 & 1.000 & 1.000 & 0.000 & 0.000 \\
\hline Setchell \& Wickings 2005, Table 3 & 11 & 0.000 & 0.968 & 0.872 & 0.867 & 0.895 & 0.000 & 0.000 \\
\hline Smith 1976, Table 1 & 7 & 0.048 & 1.000 & 1.000 & 0.886 & 0.929 & 0.008 & 0.004 \\
\hline Somers \& Nel 1998, Table 2a & 6 & 0.133 & 1.000 & 1.000 & 0.550 & 0.741 & 0.187 & 0.074 \\
\hline Somers \& Nel 1998, Table 2b & 8 & 0.107 & 0.938 & 0.750 & 0.571 & 0.691 & 0.028 & 0.019 \\
\hline Somers \& Nel 1998, Table 2c & 9 & 0.111 & 1.000 & 1.000 & 0.857 & 0.900 & 0.000 & 0.000 \\
\hline Tamm 1977, Table 1b & 10 & 0.111 & 0.951 & 0.802 & 0.625 & 0.728 & 0.003 & 0.000 \\
\hline Tarvin \& Woolfenden 1997, Table 5 & 16 & 0.292 & 0.986 & 0.946 & 0.620 & 0.684 & 0.000 & 0.000 \\
\hline Vervaecke et al. 2000, Table 2 & 6 & 0.067 & 1.000 & 1.000 & 0.900 & 0.943 & 0.038 & 0.043 \\
\hline deWaal \& Luttrell 1985, Table 1 & 24 & 0.116 & 0.998 & 0.992 & 0.819 & 0.840 & 0.000 & 0.000 \\
\hline Watt 1986, Figure $1 \mathrm{a}$ & 10 & 0.022 & 0.982 & 0.929 & 0.883 & 0.915 & 0.000 & 0.000 \\
\hline Watt 1986, Figure 1b & 10 & 0.022 & 1.000 & 1.000 & 1.000 & 1.000 & 0.000 & 0.000 \\
\hline Watt 1986, Figure 1c & 10 & 0.000 & 0.950 & 0.800 & 0.800 & 0.855 & 0.000 & 0.000 \\
\hline Watt 1986, Figure 1d & 10 & 0.000 & 0.908 & 0.633 & 0.633 & 0.733 & 0.001 & 0.000 \\
\hline Watt 1986, Figure 1e & 10 & 0.022 & 0.982 & 0.929 & 0.833 & 0.878 & 0.000 & 0.000 \\
\hline West-Eberhard 1986, Table 5 & 7 & 0.524 & 1.000 & 1.000 & 0.229 & 0.498 & 0.293 & 0.333 \\
\hline Wittemyer \& Getz 2007, Table 1 & 20 & 0.626 & 0.969 & 0.875 & 0.119 & 0.243 & 0.082 & 0.000 \\
\hline Wittig \& Boesch 2003, Figure 1 & 15 & 0.162 & 0.996 & 0.985 & 0.754 & 0.799 & 0.000 & 0.000 \\
\hline Zine \& Krausman 2000, Table 1 & 12 & 0.091 & 0.980 & 0.919 & 0.832 & 0.864 & 0.000 & 0.000 \\
\hline
\end{tabular}




\section{References}

Allee, W. C. \& Dickinson, J. C. 1954. Dominance and subordination in the smooth dogfish Mustelus canis (Mitchill). Physiological Zoology, 27, 356-364.

Appleby, M. C. 1983. The probability of linearity in hierarchies. Animal Behaviour, 31, 600-608.

Archie, E. A., Morrison, T. A., Foley, C. A. H., Moss, C. J. \& Alberts, S. C. 2006. Dominance rank relationships among wild female African elephants, Loxodonta africana. Animal Behaviour, 71, 117-127.

Barrette, C. \& Vandal, D. 1986. Social rank, dominance, antler size, and access to food in snow-bound wild woodland caribou. Behaviour, 97, 118-146.

Berman, C. M., Ionica, C. I. \& Li, J. 2004. Dominance style among Macaca thibetana on Mt. Huangshan, China. International Journal of Primatology, 25, 1283-1312.

Bonanni, R., Cafazzo, S., Fantini, C., Pontier, D. \& Natoli, E. 2007. Feeding-order in an urban feral domestic cat colony: relationship to dominance rank, sex and age. Animal Behaviour, 74, 1369-1379.

Cafazzo, S., Valsecchi, P., Bonanni, R. \& Natoli, E. 2010. Dominance in relation to age, sex, and competitive contexts in a group of free-ranging domestic dogs. Behavioral Ecology, 21, 443-455.

Cheney, D. L. 1977. The acquisition of rank and the development of reciprocal alliances among free-ranging immature baboons. Behavioral Ecology and Sociobiology, 2, 303-318.

Clutton-Brock, T. H., Greenwood, P. J. \& Powell, R. P. 1976. Ranks and relationships in highland ponies and highland cows. Zeitschrift für Tierpsychologie, 41, 202-216.

Collias, N. E. \& Taber, R. D. 1951. A field study of some grouping and dominance relations in ring-necked pheasants. Condor, 53, 265-275.

Côté, S. D. 2000. Dominance hierarchies in female mountain goats: stability, aggressiveness and determinants of rank. Behaviour, 137, 1541-1566.

Farentinos, R. C. 1972. Social dominance and mating activity in the tassel-eared squirrel (Sciurus aberti ferreus). Animal Behaviour, 20, 316-326.

Fournier, F. \& Festa-Bianchet, M. 1995. Social dominance in adult female mountain goats. Animal Behaviour, 49, 1449-1459.

Frank, L. G. 1986. Social organization of the spotted hyaena Crocuta crocuta. II. Dominance and reproduction. Animal Behaviour, 34, 1510-1527.

Handcock, M., Hunter, D., Butts, C., Goodreau, S. \& Morris, M. 2003. Statnet: Software Tools for the Statistical Modeling of Network Data. http://statnetproject.org.

Hartzler, J. E. 1970. Winter dominance relationship in black-capped chickadees. Wilson Bulletin, 82, 427-434.

Hass, C. C. \& Jenni, D. A. 1991. Structure and ontogeny of dominance relationships among bighorn rams. Canadian Journal of Zoology, 69, 471-476.

Heitor, F. \& Vicente, L. 2010. Dominance relationships and patterns of aggression in a bachelor group of Sorraia horses (Equus caballus). Journal of Ethology, 28, 35-44.

Heitor, F., do Mar Oom, M. \& Vicente, L. 2006. Social relationships in a herd of Sorraia horses: Part I. Correlates of social dominance and contexts of aggression. Behavioural Processes, 73, 170-177. 
Hewitt, S. E., McDonald, D. W. \& Dugdale, H. L. 2009. Context-dependent linear dominance hierarchies in social groups of European badgers, Meles meles. Animal Behaviour, 77, 161-169.

Hirotani, A. 1994. Dominance rank, copulatory behaviour and estimated reproductive success in male reindeer. Animal Behaviour, 48, 929-936.

Isbell, L. A. \& Pruet, J. D. 1998. Differences between vervets (Cercopithecus aethiops) and patas monkeys (Erythrocebus patas) in agonistic interactions between adult females. International Journal of Primatology, 19, 837-855.

Izar, P., Ferreira, R. G. \& Sato, T. 2006. Describing the organization of dominance relationships by dominancedirected tree method. American Journal of Primatology, 68, 189-207.

Koenig, A., Larney, E., Lu, A. \& Borries, C. 2004. Agonistic behavior and dominance relationships in female Phayre's leaf monkeys: preliminary results. American Journal of Primatology, 64, 351-357.

Lahti, K., Koivula, K. \& Orell, M. 1994. Is the social hierarchy always linear in tits? Journal of Avian Biology, 25, 347-348.

Lu, A., Koenig, A. \& Borries, C. 2008. Formal submission, tolerance and socioecological models: a test with female Hanuman langurs. Animal Behaviour, 76, 415-428.

McMahan, C. A. \& Morris, M. D. 1984. Application of maximum likelihood paired comparison ranking to estimation of a linear dominance hierarchy in animal societies. Animal Behaviour, 32, 374-378.

Marler, P. 1957. Studies of fighting in chaffinches. (1) Behaviour in relation to the social hierarchy. British Journal of Animal Behaviour, 5, 29-37.

Masure, R. H. \& Allee, W. C. 1934. The social order in flocks of the common chicken and the pigeon. Auk, 51, 306-327.

Møller, A. P. 1987. Variation in badge size in male house sparrows Passer domesticus: evidence for status signalling. Animal Behaviour, 35, 1637-1644.

Monnin T. \& Peeters, C. 1999. Dominance hierarchy and reproductive conflicts among subordinates in a monogynous queenless ant. Behavioral Ecology, 10, 323-332.

Murray, C. M. 2007. Method for assigning categorical rank in female Pan troglodytes schweinfurthii via the frequency of approaches. International Journal of Primatology, 28, 853-864.

Nakano, S. 1994. Variation in agonistic encounters in a dominance hierarchy of freely interacting red-spotted masu salmon (Oncorhynchus masou ishikawai). Ecology of Freshwater Fish, 3, 153-158.

Natoli, E. \& De Vito, E. 1991. Agonistic behaviour, dominance rank and copulatory success in a large multi-male feral cat, Felis catus L., colony in central Rome. Animal Behaviour, 42, 227-241.

Owens, D. \& Owens, M. 1996. Social dominance and reproductive patterns in brown hyaenas, Hyaena brunnea, of the central Kalahari desert. Animal Behaviour, 51, 535-551.

Paoli, T., Paligi, E. \& Borgognini Tarli, S. M. 2005. Reevaluation of dominance hierarchy in bonobos (Pan paniscus). American Journal of Physical Anthropology, 130, 116-122.

Parsons, J. \& Baptista, L. F. 1980. Crown color and dominance in the white-crowned sparrow. Auk, 4, 807-815.

Patterson, I. J. 1977. Aggression and dominance in winter flocks of shelduck Tadorna tadorna (L.). Animal Behaviour, 25, 447-459. 
Poisbleau, M., Jenouvrier, S. \& Fritz, H. 2006. Assessing the reliability of dominance scores for assigning individual ranks in a hierarchy. Animal Behaviour, 72, 835-842.

R Development Core Team 2009. R: a Language and Environment for Statistical Computing. Vienna: $R$ Foundation for Statistical Computing. http://www.R-project.org.

Richter, C., Mevis, L., Malaivijtnond, S., Schülk, O. \& Ostner, J. 2009. Social relationships in free-ranging male Macaca arctoides. International Journal of Primatology, 30, 625-642.

Robbins, M. M. 2008. Feeding competition and agonistic relationships among Bwindi Gorilla beringei. International Journal of Primatology, 29, 999-1018.

Rutberg, A. T. 1986. Dominance and its fitness consequences in American bison cows. Behaviour, 96, 62-91.

Satoh, A. \& Ohkawara, K. 2008. Dominance hierarchies and aggressive behavior among queens of the inquiline ant Vollenhovia nipponica. Insectes Sociaux, 55, 200-206.

Setchell, J. M. \& Wickings, E. J. 2005. Dominance, status signals and coloration in male mandrills (Mandrillus sphinx). Ethology, 111, 25-50.

Smith, S. M. 1976. Ecological aspects of dominance hierarchies in black-capped chickadees. Auk, 93, 95-107.

Somers, M. J. \& Nel, J. A. J. 1998. Dominance and population structure of freshwater crabs (Potamonautes perlatus Milne Edwards). South African Journal of Zoology, 33, 31-36.

Tamm, S. 1977. Social dominance in captive jackdaws (Corvus monedula). Behavioural Processes, 2, 293-299.

Tarvin, K. A. \& Woolfenden, G. E. 1997. Patterns of dominance and aggressive behavior in blue jays at a feeder. Condor, 2, 434-444.

Vervaecke, H., de Vries, H. \& van Elsacker, L. 2000. Dominance and its behavioral measures in a captive group of bonobos (Pan paniscus). International Journal of Primatology, 21, 47-68.

de Waal, F. B. M. \& Luttrell, L. M. 1985. The formal hierarchy of rhesus macaques: an investigation of the baredteeth display. American Journal of Primatology, 9, 73-85.

Watt, D. J. 1986. Relationship of plumage variability, size and sex to social dominance in Harris' sparrows. Animal Behaviour, 34, 16-27.

West-Eberhard, M. J. 1986. Dominance relations in Polistes canadensis (L.), a tropical social wasp. Monitore Zoologico Italiano, 20, 263-281.

Wittemyer, G. \& Getz, W. M. 2007. Hierarchical dominance structure and social organization in African elephants, Loxodonta africana. Animal Behaviour,73, 671-681.

Wittig, R. M. \& Boesch, C. 2003. Food competition and linear dominance hierarchy among female chimpanzees of the Taï National Park. International Journal of Primatology, 24, 847-867.

Zine, M. J. \& Krausman, P. R. 2000. Behavior of captive mountain sheep in a Mojave Desert environment. Southwestern Naturalist, 45, 184-195. 